MATHEMATICS OF COMPUTATION

Volume 67, Number 224, October 1998, Pages 1533-1563

S $0025-5718(98) 00985-5$

\title{
VECTOR SUBDIVISION SCHEMES AND MULTIPLE WAVELETS
}

\author{
RONG-QING JIA, S. D. RIEMENSCHNEIDER, AND DING-XUAN ZHOU
}

Abstract. We consider solutions of a system of refinement equations written in the form

$$
\phi=\sum_{\alpha \in \mathbb{Z}} a(\alpha) \phi(2 \cdot-\alpha),
$$

where the vector of functions $\phi=\left(\phi^{1}, \ldots, \phi^{r}\right)^{T}$ is in $\left(L_{p}(\mathbb{R})\right)^{r}$ and $a$ is a finitely supported sequence of $r \times r$ matrices called the refinement mask. Associated with the mask $a$ is a linear operator $Q_{a}$ defined on $\left(L_{p}(\mathbb{R})\right)^{r}$ by $Q_{a} f:=\sum_{\alpha \in \mathbb{Z}} a(\alpha) f(2 \cdot-\alpha)$. This paper is concerned with the convergence of the subdivision scheme associated with $a$, i.e., the convergence of the sequence $\left(Q_{a}^{n} f\right)_{n=1,2, \ldots}$ in the $L_{p}$-norm.

Our main result characterizes the convergence of a subdivision scheme associated with the mask $a$ in terms of the joint spectral radius of two finite matrices derived from the mask. Along the way, properties of the joint spectral radius and its relation to the subdivision scheme are discussed. In particular, the $L_{2}$-convergence of the subdivision scheme is characterized in terms of the spectral radius of the transition operator restricted to a certain invariant subspace. We analyze convergence of the subdivision scheme explicitly for several interesting classes of vector refinement equations.

Finally, the theory of vector subdivision schemes is used to characterize orthonormality of multiple refinable functions. This leads us to construct a class of continuous orthogonal double wavelets with symmetry.

\section{INTRODUCTION}

We are concerned with the system of refinement equations

$$
\phi^{j}=\sum_{\alpha \in \mathbb{Z}} \sum_{k=1}^{r} a_{j k}(\alpha) \phi^{k}(2 \cdot-\alpha), \quad j=1, \ldots, r,
$$

where $a_{j k}(1 \leq j, k \leq r)$ are finitely supported sequences on $\mathbb{Z}$, and $\phi^{1}, \ldots, \phi^{r}$ are the unknown functions on $\mathbb{R}$. As usual, the transpose of a matrix $A$ is denoted by $A^{T}$. We write $\phi$ for the vector $\left(\phi^{1}, \ldots, \phi^{r}\right)^{T}$ and, for each $\alpha \in \mathbb{Z}$, write $a(\alpha)$ for the $r \times r$ matrix $\left(a_{j k}(\alpha)\right)_{1 \leq j, k \leq r}$. Then (1.1) can be rewritten as

$$
\phi=\sum_{\alpha \in \mathbb{Z}} a(\alpha) \phi(2 \cdot-\alpha) .
$$

The sequence $a$ of matrices is called the refinement mask.

Received by the editor December 12, 1996.

1991 Mathematics Subject Classification. Primary 39B12, 41A25, 42C15, 65F15.

Key words and phrases. Refinement equations, multiple refinable functions, multiple wavelets, vector subdivision schemes, joint spectral radii, transition operators.

Research supported in part by NSERC Canada under Grants \# OGP 121336 and A7687. 
Taking the Fourier transform of both sides of (1.2), we obtain

$$
\hat{\phi}(\xi)=H(\xi / 2) \hat{\phi}(\xi / 2), \quad \xi \in \mathbb{R},
$$

where

$$
H(\xi):=\sum_{\alpha \in \mathbb{Z}} a(\alpha) e^{-i \alpha \xi} / 2, \quad \xi \in \mathbb{R}
$$

Let

$$
M:=H(0)=\sum_{\alpha \in \mathbb{Z}} a(\alpha) / 2 .
$$

Evidently, $H$ is $2 \pi$-periodic. In particular, $H(2 k \pi)=H(0)=M$ for all $k \in \mathbb{Z}$.

If $\phi^{1}, \ldots, \phi^{r}$ are compactly supported functions in $L_{p}(\mathbb{R})(1 \leq p \leq \infty)$, then there exist compactly supported functions $\psi^{1}, \ldots, \psi^{s}(s \leq r)$ in $L_{p}(\mathbb{R})$ having linearly independent shifts such that $\psi^{1}, \ldots, \psi^{s}$ generate the same shift-invariant space as do $\phi^{1}, \ldots, \phi^{r}$ (see [17]). If, in addition, $\phi:=\left(\phi^{1}, \ldots, \phi^{r}\right)^{T}$ is refinable with a finitely supported mask, then so is $\psi:=\left(\psi^{1}, \ldots, \psi^{s}\right)^{T}$. Since the shifts of $\psi^{1}, \ldots, \psi^{s}$ are linearly independent, they are stable (see [18]). Thus, without loss of any generality, we may assume that the shifts of $\phi^{1}, \ldots, \phi^{r}$ are stable. Note that the shifts of $\phi^{1}, \ldots, \phi^{r}$ are stable if and only if, for any $\xi \in \mathbb{R}$, the sequences $\left(\hat{\phi}^{j}(\xi+2 k \pi)\right)_{k \in \mathbb{Z}}, j=1, \ldots, r$, are linearly independent (see [18]).

If $\phi^{1}, \ldots, \phi^{r}$ are functions in $L_{1}(\mathbb{R})$ with stable shifts, it was proved by Dahmen and Micchelli [5] that the matrix $M$ has a simple eigenvalue 1 and all the other eigenvalues of $M$ are less than 1 in modulus. In fact, this result is valid under a weaker condition that the sequences $\left(\hat{\phi}^{j}(2 k \pi)\right)_{k \in \mathbb{Z}}, j=1, \ldots, r$, are linearly independent. Indeed, for $k \in \mathbb{Z}$, it follows from the refinement equation in (1.3) that

$$
\hat{\phi}\left(2^{n+1} k \pi\right)=M^{n} \hat{\phi}(2 k \pi), \quad n=1,2, \ldots .
$$

Since $\phi^{1}, \ldots, \phi^{r}$ lie in $L_{1}(\mathbb{R})$, by the Riemann-Lebesgue lemma we have

$$
\lim _{n \rightarrow \infty} M^{n} \hat{\phi}(2 k \pi)=\lim _{n \rightarrow \infty} \hat{\phi}\left(2^{n+1} k \pi\right)=0 \quad \forall k \in \mathbb{Z} \backslash\{0\} .
$$

By the assumption, the sequences $\left(\hat{\phi}^{j}(2 k \pi)\right)_{k \in \mathbb{Z}}, j=1, \ldots, r$, are linearly independent. If we denote by $\mathbb{C}^{r}$ the linear space of all $r \times 1$ vectors of complex numbers, then the vectors $\left(\hat{\phi}^{1}(2 k \pi), \ldots, \hat{\phi}^{r}(2 k \pi)\right)^{T}(k \in \mathbb{Z})$ span the space $\mathbb{C}^{r}$. Let $V$ be the linear subspace of $\mathbb{C}^{r}$ spanned by $\left(\hat{\phi}^{1}(2 k \pi), \ldots, \hat{\phi}^{r}(2 k \pi)\right)^{T}, k \in \mathbb{Z} \backslash\{0\}$. If $\left(\hat{\phi}^{1}(0), \ldots, \hat{\phi}^{r}(0)\right)^{T}=0$, then $V=\mathbb{C}^{r}$, and (1.5) tells us that the spectral radius of $M$ is less than 1 ; hence $\phi$ is identically zero. If the vector $\left(\hat{\phi}^{1}(0), \ldots, \hat{\phi}^{r}(0)\right)^{T} \neq 0$, then it is an eigenvector of $M$ corresponding to the eigenvalue 1 . In this case, $V$ has dimension $r-1$ and is invariant under $M$. Therefore, 1 is a simple eigenvalue of $M$ and the other eigenvalues of $M$ are less than 1 in modulus.

From the above discussion we may assume that the $r \times r$ matrix $M$ has the following form:

$$
M=\left[\begin{array}{ll}
1 & 0 \\
0 & \Lambda
\end{array}\right] \quad \text { and } \quad \lim _{n \rightarrow \infty} \Lambda^{n}=0 .
$$

For $j=1, \ldots, r$, we use $e_{j}$ to denote the $j$ th column of the $r \times r$ identity matrix. Obviously, $e_{1}^{T} M=e_{1}^{T}$.

Under the conditions in (1.6), it was proved by Heil and Colella in [12] that there exists a unique vector $\phi$ of compactly supported distributions such that $\phi$ satisfies 
the refinement equation $(1.2)$ and $\hat{\phi}(0)=(1,0, \ldots, 0)^{T}$. We call such a solution the normalized solution of (1.2). If $\psi$ is another distributional solution of (1.2), then we must have $\psi=c \phi$ for some constant $c$.

In order to solve the refinement equation (1.2), we introduce the linear operator $Q_{a}$ on $\left(L_{p}(\mathbb{R})\right)^{r}(1 \leq p \leq \infty)$ as follows:

$$
Q_{a} f:=\sum_{\alpha \in \mathbb{Z}} a(\alpha) f(2 \cdot-\alpha), \quad f \in\left(L_{p}(\mathbb{R})\right) .
$$

If $\phi$ is a fixed point of $Q_{a}$, i.e., $Q_{a} \phi=\phi$, then $\phi$ is a solution of the refinement equation (1.2).

Suppose $f$ is an $r \times 1$ initial vector of compactly supported functions in $L_{p}(\mathbb{R})$ such that $Q_{a}^{n} f$ converges to the normalized solution $\phi$ of (1.2) in the $L_{p}$-norm $(1 \leq p \leq \infty)$. It will be proved in Section 2 that $f$ satisfies the following moment conditions of order 1 :

$$
e_{1}^{T} \hat{f}(0)=1 \quad \text { and } \quad e_{1}^{T} \hat{f}(2 k \pi)=0 \quad \forall k \in \mathbb{Z} \backslash\{0\} .
$$

Thus, we say that the subdivision scheme associated with the mask a converges in the $L_{p}$-norm $(1 \leq p \leq \infty)$ if there exists some $\phi \in\left(L_{p}(\mathbb{R})\right)^{r}$ such that, for every compactly supported vector $f \in\left(L_{p}(\mathbb{R})\right)^{r}$ satisfying the moment conditions of order 1 ,

$$
\lim _{n \rightarrow \infty}\left\|Q_{a}^{n} f-\phi\right\|_{p}=0
$$

If this is the case, then the limit vector $\phi$ is the normalized solution of the refinement equation (1.2). In particular, if the initial vector $f$ is chosen to be a vector of continuous functions, then in the case $p=\infty, \phi$ is the uniform limit of a sequence of vectors of continuous functions, and therefore, is continuous.

Suppose $1 \leq q \leq p \leq \infty$. If the subdivision scheme converges in the $L_{p}$-norm, then it also converges in the $L_{q}$-norm.

The paper is organized as follows. In Section 2, we provide a simple necessary condition on the mask for the $L_{p}$-convergence of the associated subdivision scheme. In Section 3, we discuss the relationship between stability and convergence. In particular, it is shown that if there is a stable $L_{p}$-solution of the refinement equation, then the associated subdivision scheme converges in the $L_{p}$-norm. In Section 4 , two matrices associated with the mask of the refinement equation are introduced and properties of their joint spectral radius are studied. In Section 5, we establish our main result which characterizes the $L_{p}$-convergence of a subdivision scheme in terms of the $p$-norm joint spectral radius of the two finite matrices derived from the associated mask. In Section 6, we analyze convergence of the subdivision scheme explicitly for several interesting classes of vector refinement equations which contain some isolated examples in the literature. In Section 7 , a characterization of the $L_{2^{-}}$ convergence of the subdivision scheme is given in terms of the spectral radius of the transition operator restricted to a certain invariant subspace. Finally, in Section 8 , we apply the theory to the construction of orthogonal multiple wavelets with symmetry.

\section{Subdivision SCHEMES}

For $1 \leq p \leq \infty$, let $\left(L_{p}(\mathbb{R})\right)^{r}$ denote the linear space of all vectors $f=$ $\left(f^{1}, \ldots, f^{r}\right)^{T}$ such that $f^{1}, \ldots, f^{r} \in L_{p}(\mathbb{R})$. The norm on $\left(L_{p}(\mathbb{R})\right)^{r}$ is defined 
by

$$
\|f\|_{p}:=\left(\sum_{j=1}^{r}\left\|f^{j}\right\|_{p}^{p}\right)^{1 / p}, \quad f=\left(f^{1}, \ldots, f^{r}\right)^{T} \in\left(L_{p}(\mathbb{R})\right)^{r} .
$$

In what follows, we use $\omega(f, h)_{p}$ to denote the $L_{p}$-modulus of continuity of $f$ :

$$
\omega(f, h)_{p}:=\sup _{|t| \leq h}\|f-f(\cdot-t)\|_{p}, \quad h>0 .
$$

Suppose $f$ is an $r \times 1$ initial vector of compactly supported functions in $L_{p}(\mathbb{R})$ such that $Q_{a}^{n} f$ converges to the normalized solution $\phi$ of (1.2) in the $L_{p}$-norm $(1 \leq p \leq \infty)$. Let us show that $f$ satisfies the moment conditions of order 1 . To verify (1.8), we argue as follows. Let $f_{n}:=Q_{a}^{n} f$. It follows from (1.7) that

$$
\widehat{Q_{a} f}(\xi)=H(\xi / 2) \hat{f}(\xi / 2), \quad \xi \in \mathbb{R} .
$$

Iterating this relation $n$ times, we obtain

$$
\hat{f}_{n}(\xi)=H(\xi / 2) \cdots H\left(\xi / 2^{n}\right) \hat{f}\left(\xi / 2^{n}\right), \quad \xi \in \mathbb{R} .
$$

In particular,

$$
\hat{f}_{n}\left(2^{n+1} k \pi\right)=M^{n} \hat{f}(2 k \pi) \quad \text { for } k \in \mathbb{Z} \text { and } n=1,2, \ldots,
$$

and consequently,

$$
e_{1}^{T} \hat{f}_{n}\left(2^{n+1} k \pi\right)=e_{1}^{T} M^{n} \hat{f}(2 k \pi)=e_{1}^{T} \hat{f}(2 k \pi) .
$$

Since $\left\|f_{n}-\phi\right\|_{p} \rightarrow 0$ as $n \rightarrow \infty$, we have $\left\|f_{n}-\phi\right\|_{1} \rightarrow 0$ as $n \rightarrow \infty$, and so

$$
\lim _{n \rightarrow \infty} \hat{f}_{n}\left(2^{n+1} k \pi\right)=\lim _{n \rightarrow \infty} \hat{\phi}\left(2^{n+1} k \pi\right)= \begin{cases}\hat{\phi}(0) & \text { for } k=0, \\ 0 & \text { for } k \in \mathbb{Z} \backslash\{0\},\end{cases}
$$

by the Riemann-Lebesgue lemma. Consequently,

$$
e_{1}^{T} \hat{f}(0)=\lim _{n \rightarrow \infty} e_{1}^{T} \hat{f}_{n}(0)=e_{1}^{T} \hat{\phi}(0)=1,
$$

and

$$
e_{1}^{T} \hat{f}(2 k \pi)=\lim _{n \rightarrow \infty} e_{1}^{T} \hat{f}_{n}\left(2^{n+1} k \pi\right)=0 \quad \forall k \in \mathbb{Z} \backslash\{0\} .
$$

The preceding discussion tells us that a compactly supported $f$ must satisfy the moment conditions of order 1 if $Q_{a}^{n} f$ converges to the normalized solution $\phi$ of (1.2) in the $L_{p}$-norm $(1 \leq p \leq \infty)$.

Suppose the normalized solution $\phi$ of $(1.2)$ lies in $\left(L_{1}(\mathbb{R})\right)^{r}$. If we choose $f$ to be $\phi$, then $Q_{a}^{n} \phi=\phi$ for $n=1,2, \ldots$ Thus, by what has been proved, $\phi$ satisfies the moment conditions of order 1.

By using the Poisson summation formula, we see that (1.8) is equivalent to the following condition:

$$
\sum_{\alpha \in \mathbb{Z}} e_{1}^{T} f(\cdot-\alpha)=1
$$

The following theorem gives a necessary condition for convergence of subdivision schemes. 
Theorem 2.1. Let $a: \mathbb{Z} \rightarrow \mathbb{C}^{r \times r}$ be a finitely supported sequence of $r \times r$ matrices such that the matrix $M:=\sum_{\alpha \in \mathbb{Z}} a(\alpha) / 2$ satisfies (1.6). If the subdivision scheme associated with a converges in the $L_{p}$-norm for some $p, 1 \leq p \leq \infty$, then

$$
e_{1}^{T} \sum_{\beta \in \mathbb{Z}} a(2 \beta)=e_{1}^{T} \sum_{\beta \in \mathbb{Z}} a(2 \beta+1)=e_{1}^{T} .
$$

Proof. Let $f$ be an $r \times 1$ vector of compactly supported functions in $L_{p}(\mathbb{R})$ satisfying (2.1). If the subdivision scheme associated with $a$ converges in the $L_{p}$-norm, then $Q_{a}^{n}\left(Q_{a} f\right)$ converges in the $L_{p}$-norm. Hence, by the preceding discussion, we must have

$$
e_{1}^{T} \sum_{\alpha \in \mathbb{Z}} Q_{a} f(\cdot-\alpha)=1
$$

It follows from (1.7) that

$$
\sum_{\alpha \in \mathbb{Z}} Q_{a} f(\cdot-\alpha)=\sum_{\alpha \in \mathbb{Z}} \sum_{\beta \in \mathbb{Z}} a(\beta) f(2 \cdot-2 \alpha-\beta)=\sum_{\beta \in \mathbb{Z}} \sum_{\alpha \in \mathbb{Z}} a(\beta-2 \alpha) f(2 \cdot-\beta) .
$$

We choose $f$ to be $y \chi$, where $y$ is an $r \times 1$ vector of complex numbers with $e_{1}^{T} y=1$, and $\chi$ is the characteristic function of the unit interval $[0,1)$. Then $f$ satisfies the moment conditions of order 1 ; hence $Q_{a} f$ also satisfies the moment conditions of order 1 . For $0 \leq x<1 / 2$, we have $f(2 x)=y$ and $f(2 x-\beta)=0$ for all $\beta \in \mathbb{Z} \backslash\{0\}$. Thus, it follows from (2.4) that

$$
\sum_{\alpha \in \mathbb{Z}}\left(Q_{a} f\right)(x-\alpha)=\sum_{\alpha \in \mathbb{Z}} a(-2 \alpha) y, \quad 0 \leq x<1 / 2 .
$$

This in connection with $(2.3)$ gives

$$
e_{1}^{T} \sum_{\alpha \in \mathbb{Z}} a(-2 \alpha) y=1 .
$$

Since this relation is valid for every vector $y \in \mathbb{C}^{r}$ with $e_{1}^{T} y=1$, we conclude that

$$
e_{1}^{T} \sum_{\alpha \in \mathbb{Z}} a(-2 \alpha)=e_{1}^{T}
$$

This together with (1.6) yields

$$
e_{1}^{T} \sum_{\alpha \in \mathbb{Z}} a(1-2 \alpha)=e_{1}^{T}
$$

The proof of the theorem is complete.

In the scalar case $(r=1)$, this result was established by Cavaretta, Dahmen, and Micchelli [1] for the case $p=\infty$, and by Jia [16] for the general case $1 \leq p \leq \infty$.

\section{Stability}

Let $\phi^{1}, \ldots, \phi^{r}$ be compactly supported functions in $L_{p}(\mathbb{R})(1 \leq p \leq \infty)$. It is known (see [18]) that there exists a constant $C_{1}>0$ such that

$$
\left\|\sum_{j=1}^{r} \sum_{\alpha \in \mathbb{Z}} b_{j}(\alpha) \phi^{j}(\cdot-\alpha)\right\|_{p} \leq C_{1} \sum_{j=1}^{r}\left\|b_{j}\right\|_{p} \quad \forall b_{j} \in \ell_{p}(\mathbb{Z}), j=1, \ldots, r .
$$


We say that the shifts of $\phi^{1}, \ldots, \phi^{r}$ are stable, if there exists a constant $C_{2}>0$ such that

$$
\left\|\sum_{j=1}^{r} \sum_{\alpha \in \mathbb{Z}} b_{j}(\alpha) \phi^{j}(\cdot-\alpha)\right\|_{p} \geq C_{2} \sum_{j=1}^{r}\left\|b_{j}\right\|_{p} \quad \forall b_{j} \in \ell_{p}(\mathbb{Z}), j=1, \ldots, r .
$$

It was proved by Jia and Micchelli in [18] that the shifts of the functions $\phi^{1}, \ldots, \phi^{r}$ are stable if and only if, for any $\xi \in \mathbb{R}$, the sequences $\left(\hat{\phi}^{j}(\xi+2 \pi \beta)\right)_{\beta \in \mathbb{Z}}(j=1, \ldots, r)$ are linearly independent.

For $y=\left(y_{1}, \ldots, y_{r}\right)^{T} \in \mathbb{C}^{r}$, we define

$$
\|y\|_{p}:= \begin{cases}\left(\sum_{j=1}^{r}\left|y_{j}\right|^{p}\right)^{1 / p} & \text { for } 1 \leq p<\infty \\ \max _{1 \leq j \leq r}\left|y_{j}\right| & \text { for } p=\infty\end{cases}
$$

We denote by $\ell_{p}\left(\mathbb{Z} \rightarrow \mathbb{C}^{r}\right)$ the linear space of all sequences $u: \mathbb{Z} \rightarrow \mathbb{C}^{r}$ such that $u(\alpha)=\left(u_{1}(\alpha), \ldots, u_{r}(\alpha)\right)^{T}$ for some $u_{1}, \ldots, u_{r} \in \ell_{p}(\mathbb{Z})$ and for all $\alpha \in \mathbb{Z}$. Obviously, $u \mapsto\left(u_{1}, \ldots, u_{r}\right)^{T}$ is a canonical isomorphism between $\ell_{p}\left(\mathbb{Z} \rightarrow \mathbb{C}^{r}\right)$ and $\left(\ell_{p}(\mathbb{Z})\right)^{r}$. Thus, we may identify $\ell_{p}\left(\mathbb{Z} \rightarrow \mathbb{C}^{r}\right)$ with $\left(\ell_{p}(\mathbb{Z})\right)^{r}$. The norm of $u=\left(u_{1}, \ldots, u_{r}\right)^{T}$ is given by

$$
\|u\|_{p}:=\left(\sum_{j=1}^{r}\left\|u_{j}\right\|_{p}^{p}\right)^{1 / p}
$$

Equipped with this norm, $\left(\ell_{p}(\mathbb{Z})\right)^{r}$ becomes a Banach space.

We denote by $\ell_{p}\left(\mathbb{Z} \rightarrow \mathbb{C}^{r \times r}\right)$ the linear space of all matrices $b: \mathbb{Z} \rightarrow \mathbb{C}^{r \times r}$ such that $b(\alpha)=\left(b_{j k}(\alpha)\right)_{1 \leq j, k \leq r}$ for some $b_{j k} \in \ell_{p}(\mathbb{Z})(j, k=1, \ldots, r)$ and for all $\alpha \in \mathbb{Z}$. We also identify $\ell_{p}\left(\mathbb{Z} \rightarrow \mathbb{C}^{r \times r}\right)$ with $\left(\ell_{p}(\mathbb{Z})\right)^{r \times r}$. The norm of $b=\left(b_{j k}\right)_{1 \leq j, k \leq r}$ is defined by

$$
\|b\|_{p}:=\left\{\sum_{j=1}^{r} \sum_{k=1}^{r}\left\|b_{j k}\right\|_{p}^{p}\right\}^{1 / p} .
$$

Let $\phi=\left(\phi^{1}, \ldots, \phi^{r}\right)^{T}$ be a vector of compactly supported functions in $L_{p}(\mathbb{R})$. Then there exists a constant $C_{1}>0$ such that

$$
\left\|\sum_{\alpha \in \mathbb{Z}} b(\alpha) \phi(\cdot-\alpha)\right\|_{p} \leq C_{1}\|b\|_{p} \quad \forall b \in\left(\ell_{p}(\mathbb{Z})\right)^{r \times r} .
$$

If, in addition, the shifts of the functions $\phi^{1}, \ldots, \phi^{r}$ are stable, then there exists a constant $C_{2}>0$ such that

$$
\left\|\sum_{\alpha \in \mathbb{Z}} b(\alpha) \phi(\cdot-\alpha)\right\|_{p} \geq C_{2}\|b\|_{p} \quad \forall b \in\left(\ell_{p}(\mathbb{Z})\right)^{r \times r} .
$$

Let $\ell(\mathbb{Z})$ denote the linear space of all sequences on $\mathbb{Z}$, and let $\ell_{0}(\mathbb{Z})$ denote the linear space of all finitely supported sequences on $\mathbb{Z}$. Furthermore, we denote by $\ell_{0}\left(\mathbb{Z} \rightarrow \mathbb{C}^{r}\right)\left(\right.$ resp. $\left.\ell_{0}\left(\mathbb{Z} \rightarrow \mathbb{C}^{r \times r}\right)\right)$ the linear space of all finitely supported sequences of $r \times 1$ vectors (resp. $r \times r$ matrices). We identify $\ell_{0}\left(\mathbb{Z} \rightarrow \mathbb{C}^{r}\right)$ with $\left(\ell_{0}(\mathbb{Z})\right)^{r}$, and identify $\ell_{0}\left(\mathbb{Z} \rightarrow \mathbb{C}^{r \times r}\right)$ with $\left(\ell_{0}(\mathbb{Z})\right)^{r \times r}$.

Theorem 3.1. Let $a: \mathbb{Z} \rightarrow \mathbb{C}^{r \times r}$ be a finitely supported sequence of $r \times r$ matrices such that the matrix $M:=\sum_{\alpha \in \mathbb{Z}} a(\alpha) / 2$ satisfies (1.6), and let $Q=Q_{a}$ be the linear operator given by (1.7). Suppose $f=\left(f^{1}, \ldots, f^{r}\right)^{T}$ is a vector of compactly 
supported functions in $L_{p}(\mathbb{R})(1 \leq p \leq \infty)$, $f$ satisfies the moment conditions of order 1 , and the shifts of $f^{1}, \ldots, f^{r}$ are stable. If there exists a vector $\phi$ of functions in $L_{p}(\mathbb{R})$ (a vector of continuous functions in the case $p=\infty$ ) such that

$$
\lim _{n \rightarrow \infty}\left\|Q^{n} f-\phi\right\|_{p}=0
$$

then for any $r \times 1$ vector $g$ of compactly supported functions in $L_{p}(\mathbb{R})$ satisfying the moment conditions of order 1 we also have

$$
\lim _{n \rightarrow \infty}\left\|Q^{n} g-\phi\right\|_{p}=0
$$

Proof. The proof follows the lines of [16, Theorem 2.2]. For $j=1, \ldots, r$ and $n=0,1,2, \ldots$, let $\lambda_{n, j}$ be the sequence on $\mathbb{Z}$ given by

$$
\lambda_{n, j}(\alpha):=2^{n} \int_{\alpha / 2^{n}}^{(\alpha+1) / 2^{n}} \phi^{j}(x) d x, \quad \alpha \in \mathbb{Z} .
$$

In other words, $\lambda_{n, j}(\alpha)$ is the average value of $\phi^{j}$ on the interval $\left[\alpha / 2^{n},(\alpha+1) / 2^{n}\right)$. Let $b_{n} \in\left(\ell_{p}(\mathbb{Z})\right)^{r \times r}$ be given by

$$
b_{n}:=\left[\begin{array}{cccc}
\lambda_{n, 1} & 0 & \cdots & 0 \\
\lambda_{n, 2} & 0 & \cdots & 0 \\
\vdots & \vdots & \ddots & \vdots \\
\lambda_{n, r} & 0 & \cdots & 0
\end{array}\right] .
$$

Set

$$
f_{n}:=\sum_{\alpha \in \mathbb{Z}} b_{n}(\alpha) f\left(2^{n} \cdot-\alpha\right) \quad \text { and } \quad g_{n}:=\sum_{\alpha \in \mathbb{Z}} b_{n}(\alpha) g\left(2^{n} \cdot-\alpha\right) .
$$

Since $f$ and $g$ satisfy the moment conditions of order 1 , there exists a constant $C_{1}>0$ such that

$$
\left\|\phi-f_{n}\right\|_{p} \leq C_{1} \omega\left(\phi, 1 / 2^{n}\right)_{p} \quad \text { and } \quad\left\|\phi-g_{n}\right\|_{p} \leq C_{1} \omega\left(\phi, 1 / 2^{n}\right)_{p}
$$

(see, e.g., [16, Theorem 2.1]). Write

$$
Q^{n} f=\sum_{\alpha \in \mathbb{Z}} a_{n}(\alpha) f\left(2^{n} \cdot-\alpha\right) \quad \text { and } \quad Q^{n} g=\sum_{\alpha \in \mathbb{Z}} a_{n}(\alpha) g\left(2^{n} \cdot-\alpha\right),
$$

where each $a_{n}$ is an element of $\ell_{0}\left(\mathbb{Z} \rightarrow \mathbb{C}^{r \times r}\right)$. Thus, we obtain

$$
Q^{n} f-f_{n}=\sum_{\alpha \in \mathbb{Z}}\left[a_{n}(\alpha)-b_{n}(\alpha)\right] f\left(2^{n} \cdot-\alpha\right) .
$$

Since the shifts of $f^{1}, \ldots, f^{r}$ are stable, there exists a constant $C_{2}>0$ such that

$$
\left\|a_{n}-b_{n}\right\|_{p} \leq C_{2}\left\|\left(f_{n}-Q^{n} f\right)\left(2^{-n} \cdot\right)\right\|_{p}=2^{n / p} C_{2}\left\|f_{n}-Q^{n} f\right\|_{p} .
$$

Furthermore,

$$
Q^{n} g-g_{n}=\sum_{\alpha \in \mathbb{Z}}\left[a_{n}(\alpha)-b_{n}(\alpha)\right] g\left(2^{n} \cdot-\alpha\right) .
$$

Hence there exists a constant $C_{3}>0$ such that

$$
\left\|\left(g_{n}-Q^{n} g\right)\left(2^{-n} \cdot\right)\right\|_{p} \leq C_{3}\left\|a_{n}-b_{n}\right\|_{p} .
$$

Combining the above estimates, we see that there exists a constant $C>0$ such that

$$
\left\|g_{n}-Q^{n} g\right\|_{p} \leq C\left\|f_{n}-Q^{n} f\right\|_{p} .
$$


Therefore we have

$$
\begin{aligned}
\left\|\phi-Q^{n} g\right\|_{p} & \leq\left\|\phi-g_{n}\right\|_{p}+\left\|g_{n}-Q^{n} g\right\|_{p} \\
& \leq\left\|\phi-g_{n}\right\|_{p}+C\left\|f_{n}-Q^{n} f\right\|_{p} \\
& \leq\left\|\phi-g_{n}\right\|_{p}+C\left(\left\|\phi-f_{n}\right\|_{p}+\left\|\phi-Q^{n} f\right\|_{p}\right) .
\end{aligned}
$$

But as $n \rightarrow \infty,\left\|\phi-g_{n}\right\|_{p} \rightarrow 0,\left\|\phi-f_{n}\right\|_{p} \rightarrow 0$, and $\left\|\phi-Q^{n} f\right\|_{p} \rightarrow 0$; hence we conclude that

$$
\lim _{n \rightarrow \infty}\left\|Q^{n} g-\phi\right\|_{p}=0
$$

Let $\phi=\left(\phi^{1}, \ldots, \phi^{r}\right)^{T}$ be the normalized solution of the refinement equation (1.2). Then $Q_{a} \phi=\phi$. Suppose $\phi^{1}, \ldots, \phi^{r}$ lie in $L_{p}(\mathbb{R})\left(\phi^{1}, \ldots, \phi^{r}\right.$ are continuous in the case $p=\infty$ ) and the shifts of them are stable. In this case, $\phi$ must satisfy the moment conditions of order 1. Thus, in Theorem 3.1, we may choose $f$ to be $\phi$. This gives the following result.

Theorem 3.2. Let $a: \mathbb{Z} \rightarrow \mathbb{C}^{r \times r}$ be a finitely supported sequence of $r \times r$ matrices such that the matrix $M:=\sum_{\alpha \in \mathbb{Z}} a(\alpha) / 2$ satisfies (1.6), and let $\phi=\left(\phi^{1}, \ldots, \phi^{r}\right)^{T}$ be the normalized solution of the refinement equation (1.2). If $\phi^{1}, \ldots, \phi^{r}$ lie in $L_{p}(\mathbb{R})\left(\phi^{1}, \ldots, \phi^{r}\right.$ are continuous in the case $\left.p=\infty\right)$ and the shifts of them are stable, then the subdivision scheme associated with the mask a converges to $\phi$ in the $L_{p}$-norm.

In the scalar case $(r=1)$, this theorem was established by Cavaretta, Dahmen, and Micchelli [1] for the case $p=\infty$, and by Jia [16] for the general case $1 \leq p \leq \infty$.

For the scalar case $(r=1)$, Jia and Wang [21] gave a characterization for the stability and linear independence of the shifts of a refinable function in terms of the refinement mask. Their results were extended by Zhou [33] to the case where the scaling factor is an arbitrary integer greater than 1 . For the vector case $(r>1)$, stability of the shifts of multiple refinable functions was discussed by Hervé [14], Hogan [15], and Wang [32]. Assuming the vector of refinable functions lies in $\left(L_{2}(\mathbb{R})\right)^{r}$, Shen [30] gave a characterization for $L_{2}$-stability. See [23] for a related work.

\section{The Joint SPECTRAL Radius}

Let $Q_{a}$ be the linear operator given in (1.7). For an initial vector $f \in\left(L_{p}(\mathbb{R})\right)^{r}$, we have

$$
Q_{a}^{n} f=\sum_{\alpha \in \mathbb{Z}} a_{n}(\alpha) f\left(2^{n} \cdot-\alpha\right), \quad n=1,2, \ldots,
$$

where each $a_{n}$ is independent of the choice of $f$. In particular, $a_{1}=a$. Consequently, for $n>1$ we have

$$
\begin{aligned}
Q_{a}^{n} f & =Q_{a}^{n-1}\left(Q_{a} f\right)=\sum_{\beta \in \mathbb{Z}} a_{n-1}(\beta)\left(Q_{a} f\right)\left(2^{n-1} \cdot-\beta\right) \\
& =\sum_{\beta \in \mathbb{Z}} \sum_{\alpha \in \mathbb{Z}} a_{n-1}(\beta) a(\alpha) f\left(2^{n} \cdot-2 \beta-\alpha\right) \\
& =\sum_{\alpha \in \mathbb{Z}}\left[\sum_{\beta \in \mathbb{Z}} a_{n-1}(\beta) a(\alpha-2 \beta)\right] f\left(2^{n} \cdot-\alpha\right) .
\end{aligned}
$$


This establishes the following iteration relation for $a_{n}(n=1,2, \ldots)$ :

$$
a_{1}=a \quad \text { and } \quad a_{n}(\alpha)=\sum_{\beta \in \mathbb{Z}} a_{n-1}(\beta) a(\alpha-2 \beta), \quad \alpha \in \mathbb{Z} .
$$

For $\varepsilon \in \mathbb{Z}$, we denote by $A_{\varepsilon}=\left(A_{\varepsilon}(\alpha, \beta)\right)_{\alpha, \beta \in \mathbb{Z}}$ the bi-infinite block matrix given by

$$
A_{\varepsilon}(\alpha, \beta):=a(\varepsilon+2 \alpha-\beta), \quad \alpha, \beta \in \mathbb{Z} .
$$

Lemma 4.1. For $a \in\left(\ell_{0}(\mathbb{Z})\right)^{r \times r}$ and $n=1,2, \ldots$, let $a_{n} \in\left(\ell_{0}(\mathbb{Z})\right)^{r \times r}$ be given by the iteration relation (4.2). If $\alpha=\varepsilon_{1}+2 \varepsilon_{2}+\cdots+2^{n-1} \varepsilon_{n}+2^{n} \gamma$, where $\varepsilon_{1}, \ldots, \varepsilon_{n}, \gamma \in$ $\mathbb{Z}$, then

$$
a_{n}(\alpha-\beta)=A_{\varepsilon_{n}} \cdots A_{\varepsilon_{1}}(\gamma, \beta) \quad \forall \beta \in \mathbb{Z} .
$$

Proof. The proof proceeds by induction on $n$. For $n=1$ and $\alpha=\varepsilon_{1}+2 \gamma$, we have

$$
a_{1}(\alpha-\beta)=a\left(\varepsilon_{1}+2 \gamma-\beta\right)=A_{\varepsilon_{1}}(\gamma, \beta) .
$$

Suppose $n>1$ and the lemma has been verified for $n-1$. For $\alpha=\varepsilon_{1}+2 \alpha_{1}$, where $\alpha_{1}, \varepsilon \in \mathbb{Z}$, by the iteration relation (4.2) we have

$$
a_{n}(\alpha-\beta)=\sum_{\eta \in \mathbb{Z}} a_{n-1}(\eta) a(\alpha-\beta-2 \eta)=\sum_{\eta \in \mathbb{Z}} a_{n-1}\left(\alpha_{1}-\eta\right) a\left(\varepsilon_{1}+2 \eta-\beta\right) .
$$

Suppose $\alpha_{1}=\varepsilon_{2}+\cdots+2^{n-2} \varepsilon_{n}+2^{n-1} \gamma$. Then by the induction hypothesis we have

$$
a_{n-1}\left(\alpha_{1}-\eta\right)=A_{\varepsilon_{n}} \cdots A_{\varepsilon_{2}}(\gamma, \eta)
$$

This in connection with (4.4) gives

$$
a_{n}(\alpha-\beta)=\sum_{\eta \in \mathbb{Z}} A_{\varepsilon_{n}} \cdots A_{\varepsilon_{2}}(\gamma, \eta) A_{\varepsilon_{1}}(\eta, \beta)=A_{\varepsilon_{n}} \cdots A_{\varepsilon_{2}} A_{\varepsilon_{1}}(\gamma, \beta),
$$

thereby completing the induction procedure.

In the scalar case $(r=1)$, Lemma 4.1 was established by Goodman, Micchelli, and Ward [10].

Lemma 4.1 motivates us to consider the joint spectral radius of a finite collection of linear operators. The uniform joint spectral radius was introduced by Rota and Strang in [29], and the $p$-norm joint spectral radius was introduced by Jia in [16]. Let us recall from [16] the definition of the $p$-norm joint spectral radius.

Let $V$ be a finite-dimensional vector space equipped with a vector norm $\|\cdot\|$. For a linear operator $A$ on $V$, define

$$
\|A\|:=\max _{\|v\|=1}\{\|A v\|\} \text {. }
$$

Let $\mathcal{A}$ be a finite collection of linear operators on $V$. For a positive integer $n$ we denote by $\mathcal{A}^{n}$ the $n$th Cartesian power of $\mathcal{A}$ :

$$
\mathcal{A}^{n}=\left\{\left(A_{1}, \ldots, A_{n}\right): A_{1}, \ldots, A_{n} \in \mathcal{A}\right\} .
$$

For $1 \leq p<\infty$, let

$$
\left\|\mathcal{A}^{n}\right\|_{p}:=\left(\sum_{\left(A_{1}, \ldots, A_{n}\right) \in \mathcal{A}^{n}}\left\|A_{1} \cdots A_{n}\right\|^{p}\right)^{1 / p}
$$


and, for $p=\infty$, define

$$
\left\|\mathcal{A}^{n}\right\|_{\infty}:=\max \left\{\left\|A_{1} \cdots A_{n}\right\|:\left(A_{1}, \ldots, A_{n}\right) \in \mathcal{A}^{n}\right\} .
$$

For $1 \leq p \leq \infty$, the $p$-norm joint spectral radius of $\mathcal{A}$ is defined to be

$$
\rho_{p}(\mathcal{A}):=\lim _{n \rightarrow \infty}\left\|\mathcal{A}^{n}\right\|_{p}^{1 / n} .
$$

It is easily seen that this limit indeed exists, and

$$
\lim _{n \rightarrow \infty}\left\|\mathcal{A}^{n}\right\|_{p}^{1 / n}=\inf _{n \geq 1}\left\|\mathcal{A}^{n}\right\|_{p}^{1 / n} .
$$

Clearly, $\rho_{p}(\mathcal{A})$ is independent of the choice of the vector norm on $V$.

If $\mathcal{A}$ consists of a single linear operator $A$, then $\rho_{p}(\mathcal{A})=\rho(A)$, where $\rho(A)$ denotes the spectral radius of $A$, which is independent of $p$. It is easily seen that $\rho(A) \leq \rho_{\infty}(\mathcal{A})$ for any element $A$ in $\mathcal{A}$.

The above definition of joint spectral radius also applies to a finite collection of square matrices of the same size. Indeed, an $s \times s$ matrix can be viewed as a linear operator on $\mathbb{C}^{s}$. Thus, if $\mathcal{A}$ is a finite collection of $s \times s$ matrices, the joint spectral radius $\rho_{p}(\mathcal{A})$ is well defined for $1 \leq p \leq \infty$.

Suppose $\mathcal{A}=\left\{A_{1}, \ldots, A_{m}\right\}$ and each $A_{j}$ is a block triangular matrix:

$$
A_{j}=\left(\begin{array}{cc}
E_{j} & G_{j} \\
0 & F_{j}
\end{array}\right), \quad j=1, \ldots, m,
$$

where $E_{1}, \ldots, E_{m}$ are square matrices of the same size, and so are $F_{1}, \ldots, F_{m}$. In this case, we have the following result.

Lemma 4.2. If the matrices $A_{1}, \ldots, A_{m}$ are of the form (4.5), then

$$
\rho_{p}\left(A_{1}, \ldots, A_{m}\right)=\max \left\{\rho_{p}\left(E_{1}, \ldots, E_{m}\right), \rho_{p}\left(F_{1}, \ldots, F_{m}\right)\right\}, \quad 1 \leq p \leq \infty .
$$

Proof. In our proof the norm of a matrix $A$, denoted by $\|A\|$, is chosen to be its maximum absolute row sum. We write $\rho$ for the right-hand side of (4.6). It is easily seen that $\rho \leq \rho_{p}\left(A_{1}, \ldots, A_{m}\right)$. Thus, it suffices to show $\rho_{p}\left(A_{1}, \ldots, A_{m}\right) \leq \rho$.

Suppose $1 \leq \varepsilon_{1}, \ldots, \varepsilon_{n} \leq m$. By induction on $n$ we can easily derive that

$$
A_{\varepsilon_{1}} \cdots A_{\varepsilon_{n}}=\left(\begin{array}{cc}
E_{\varepsilon_{1}} \cdots E_{\varepsilon_{n}} & B_{\varepsilon_{1}, \ldots, \varepsilon_{n}} \\
0 & F_{\varepsilon_{1}} \cdots F_{\varepsilon_{n}}
\end{array}\right)
$$

where

$$
B_{\varepsilon_{1}, \ldots, \varepsilon_{n}}=\sum_{k=1}^{n} E_{\varepsilon_{1}} \cdots E_{\varepsilon_{k-1}} G_{\varepsilon_{k}} F_{\varepsilon_{k+1}} \cdots F_{\varepsilon_{n}}
$$

Let us first establish (4.6) for $p=\infty$. We have

$$
\left\|A_{\varepsilon_{1}} \cdots A_{\varepsilon_{n}}\right\| \leq \max \left\{\left\|E_{\varepsilon_{1}} \cdots E_{\varepsilon_{n}}\right\|+\left\|B_{\varepsilon_{1}, \ldots, \varepsilon_{n}}\right\|,\left\|F_{\varepsilon_{1}} \cdots F_{\varepsilon_{n}}\right\|\right\}
$$

and

$$
\left\|B_{\varepsilon_{1}, \ldots, \varepsilon_{n}}\right\| \leq \sum_{k=1}^{n}\left\|E_{\varepsilon_{1}} \cdots E_{\varepsilon_{k-1}}\right\|\left\|G_{\varepsilon_{k}}\right\|\left\|F_{\varepsilon_{k+1}} \cdots F_{\varepsilon_{n}}\right\| .
$$

Let $t$ be a fixed positive real number. By the very definition of the uniform joint spectral radius, there exists an integer $K>0$ such that

$$
\left\|E_{\eta_{1}} \cdots E_{\eta_{k}}\right\| \leq(\rho+t)^{k} \quad \text { and } \quad\left\|F_{\eta_{1}} \cdots F_{\eta_{k}}\right\| \leq(\rho+t)^{k},
$$


provided $\eta_{1}, \ldots, \eta_{k} \in\{1, \ldots, m\}$ and $k \geq K$. For $1 \leq k \leq K$ we have

$$
\left\|E_{\eta_{1}} \cdots E_{\eta_{k}}\right\| \leq C(\rho+t)^{k} \text { and }\left\|F_{\eta_{1}} \cdots F_{\eta_{k}}\right\| \leq C(\rho+t)^{k},
$$

where

$$
C:=\max \left\{\left[\left\|A_{j}\right\| /(\rho+t)\right]^{k}: j=1, \ldots, m ; k=0, \ldots, K\right\} .
$$

Note that $\left\|G_{j}\right\| \leq\left\|A_{j}\right\| \leq C(\rho+t)$ for $1 \leq j \leq m$. The above arguments tell us that

$$
\left\|B_{\varepsilon_{1}, \ldots, \varepsilon_{n}}\right\| \leq \sum_{k=1}^{n}\left\|E_{\varepsilon_{1}} \cdots E_{\varepsilon_{k-1}}\right\|\left\|G_{\varepsilon_{k}}\right\|\left\|F_{\varepsilon_{k+1}} \cdots F_{\varepsilon_{n}}\right\| \leq n C^{3}(\rho+t)^{n} .
$$

Hence

$$
\left\|A_{\varepsilon_{1}} \cdots A_{\varepsilon_{n}}\right\| \leq(\rho+t)^{n}+n C^{3}(\rho+t)^{n} \quad \text { for } n \geq K .
$$

It follows that

$$
\rho_{\infty}\left(A_{1}, \ldots, A_{m}\right) \leq \lim _{n \rightarrow \infty}\left[(\rho+t)^{n}+n C^{3}(\rho+t)^{n}\right]^{1 / n}=\rho+t .
$$

But $t>0$ can be arbitrarily small; therefore $\rho_{\infty}\left(A_{1}, \ldots, A_{m}\right) \leq \rho$, as desired.

For the case $1 \leq p<\infty$, we observe that

$$
\begin{aligned}
\sum_{1 \leq \varepsilon_{1}, \ldots, \varepsilon_{n} \leq m} & \left\|E_{\varepsilon_{1}} \cdots E_{\varepsilon_{k-1}} G_{\varepsilon_{k}} F_{\varepsilon_{k+1}} \cdots F_{\varepsilon_{n}}\right\|^{p} \\
& \leq \sum_{1 \leq \varepsilon_{1}, \ldots, \varepsilon_{n} \leq m}\left\|E_{\varepsilon_{1}} \cdots E_{\varepsilon_{k-1}}\right\|^{p}\left\|G_{\varepsilon_{k}}\right\|^{p}\left\|F_{\varepsilon_{k+1}} \cdots F_{\varepsilon_{n}}\right\|^{p} \\
& =\left[\sum_{1 \leq \varepsilon_{1}, \ldots, \varepsilon_{k-1} \leq m}\left\|E_{\varepsilon_{1}} \cdots E_{\varepsilon_{k-1}}\right\|^{p}\right]\left[\sum_{1 \leq \varepsilon_{k} \leq m}\left\|G_{\varepsilon_{k}}\right\|^{p}\right] \\
& \times\left[\sum_{1 \leq \varepsilon_{k+1}, \ldots, \varepsilon_{n} \leq m}\left\|F_{\varepsilon_{k+1}} \cdots F_{\varepsilon_{n}}\right\|^{p}\right] .
\end{aligned}
$$

The rest of the proof is similar to that for the case $p=\infty$.

Now let $\mathcal{A}$ be a finite collection of linear operators on a normed vector space $V$, which is not necessarily finite dimensional. A subspace $W$ of $V$ is said to be invariant under $\mathcal{A}$, or $\mathcal{A}$-invariant, if it is invariant under every operator $A$ in $\mathcal{A}$. For a vector $w \in V$, we define

$$
\left\|\mathcal{A}^{n} w\right\|_{p}:= \begin{cases}\left(\sum_{\left(A_{1}, \ldots, A_{n}\right) \in \mathcal{A}^{n}}\left\|A_{1} \cdots A_{n} w\right\|^{p}\right)^{1 / p} & \text { for } 1 \leq p<\infty \\ \max \left\{\left\|A_{1} \cdots A_{n} w\right\|:\left(A_{1}, \ldots, A_{n}\right) \in \mathcal{A}^{n}\right\} & \text { for } p=\infty\end{cases}
$$

If the minimal $\mathcal{A}$-invariant subspace $W$ generated by $w$ is finite dimensional, then we have

$$
\lim _{n \rightarrow \infty}\left\|\mathcal{A}^{n} w\right\|_{p}^{1 / n}=\rho_{p}\left(\left.\mathcal{A}\right|_{W}\right), \quad 1 \leq p \leq \infty .
$$

See [11, Lemma 2.4] for a proof of this result.

Let $a$ be an element of $\left(\ell_{0}(\mathbb{Z})\right)^{r \times r}$. The biinfinite block matrices $A_{\varepsilon}(\varepsilon \in \mathbb{Z})$ defined in (4.3) may be viewed as the linear operators on $\left(\ell_{0}(\mathbb{Z})\right)^{r}$ given by

$$
A_{\varepsilon} v(\alpha)=\sum_{\beta \in \mathbb{Z}} a(\varepsilon+2 \alpha-\beta) v(\beta), \quad \alpha \in \mathbb{Z}, \quad v \in\left(\ell_{0}(\mathbb{Z})\right)^{r} .
$$


For a bounded subset $K$ of $\mathbb{R}$ denote by $\ell(K)$ the subspace of $\ell_{0}(\mathbb{Z})$ consisting of all sequences supported in $K$. Suppose $a$ is supported on $[0, N]$, where $N$ is a positive integer. Then, for $j \leq 0$ and $k \geq N-1,(\ell([j, k]))^{r}$ is invariant under $A_{0}$ and $A_{1}$. Consequently, the minimal common invariant subspace of $A_{0}$ and $A_{1}$ generated by a finite subset of $\left(\ell_{0}(\mathbb{Z})\right)^{r}$ is finite dimensional.

For two elements $b$ and $c$ in $\ell_{0}(\mathbb{Z})$, the discrete convolution of $b$ and $c$, denoted by $b * c$, is the element of $\ell_{0}(\mathbb{Z})$ defined by

$$
b * c(\alpha)=\sum_{\beta \in \mathbb{Z}} b(\alpha-\beta) c(\beta), \quad \alpha \in \mathbb{Z} .
$$

If $b \in\left(\ell_{0}(\mathbb{Z})\right)^{r \times r}$ and $c \in\left(\ell_{0}(\mathbb{Z})\right)^{r}$, then $b * c \in\left(\ell_{0}(\mathbb{Z})\right)^{r}$ is defined in a similar way.

For $\beta \in \mathbb{Z}$, we denote by $\delta_{\beta}$ the sequence on $\mathbb{Z}$ given by

$$
\delta_{\beta}(\alpha)= \begin{cases}1 & \text { for } \alpha=\beta, \\ 0 & \text { for } \alpha \in \mathbb{Z} \backslash\{\beta\} .\end{cases}
$$

In particular, we write $\delta$ for $\delta_{0}$. Evidently, $b * \delta_{\beta}=b(\cdot-\beta)$ for any $b \in \ell_{0}(\mathbb{Z})$. Let $a_{n}(n=1,2, \ldots)$ be the sequences given by the iteration relation (4.2). For an $r \times 1$ vector $y \in \mathbb{C}^{r}$, we observe that $a_{n} y$ is an element in $\left(\ell_{0}(\mathbb{Z})\right)^{r}$ given by $a_{n} y(\alpha)=a_{n}(\alpha) y, \alpha \in \mathbb{Z}$. Also, $y \delta_{\beta}$ is the obvious element in $\left(\ell_{0}(\mathbb{Z})\right)^{r}$ given by $\left(y \delta_{\beta}\right)(\alpha)=y$ if $\alpha=\beta$ and $\left(y \delta_{\beta}\right)(\alpha)=0$ otherwise. Likewise, the difference operator $\nabla$ on $\ell(\mathbb{Z}), \nabla: a \mapsto \nabla a$, maps a sequence $a$ on $\mathbb{Z}$ to the sequence $\nabla a:=a(\cdot)-a(\cdot-1)$, and $y \nabla \delta_{\beta}$ is the element of $\left(\ell_{0}(\mathbb{Z})\right)^{r}$ given by $y \delta_{\beta}-y \delta_{\beta+1}$.

For the following lemma, the underlying vector norm in (4.7) is taken to be $\|\cdot\|_{p}$ for the same value of $p$.

Lemma 4.3. Let $\mathcal{A}:=\left\{A_{0}, A_{1}\right\}$ and $v \in\left(\ell_{0}(\mathbb{Z})\right)^{r}$. Then

$$
\left\|\mathcal{A}^{n} v\right\|_{p}=\left\|a_{n} * v\right\|_{p}, \quad 1 \leq p \leq \infty .
$$

Consequently, the identities

$$
\left\|\mathcal{A}^{n}\left(y \delta_{\beta}\right)\right\|_{p}=\left\|a_{n} y\right\|_{p} \quad \text { and } \quad\left\|\mathcal{A}^{n}\left(y \nabla \delta_{\beta}\right)\right\|_{p}=\left\|\nabla a_{n} y\right\|_{p}
$$

hold true for $1 \leq p \leq \infty, \beta \in \mathbb{Z}$, and $y \in \mathbb{C}^{r}$.

Proof. For $1 \leq p<\infty$ we have

$$
\begin{aligned}
\left\|a_{n} * v\right\|_{p}^{p} & =\sum_{\alpha \in \mathbb{Z}}\left\|a_{n} * v(\alpha)\right\|_{p}^{p}=\sum_{\alpha \in \mathbb{Z}}\left\|\sum_{\beta \in \mathbb{Z}} a_{n}(\alpha-\beta) v(\beta)\right\|_{p}^{p} \\
= & \sum_{\varepsilon_{1}, \ldots, \varepsilon_{n} \in\{0,1\}} \sum_{\gamma \in \mathbb{Z}}\left\|\sum_{\beta \in \mathbb{Z}} A_{\varepsilon_{n}} \cdots A_{\varepsilon_{1}} \delta_{\beta}(\gamma) v(\beta)\right\|_{p}^{p} \\
= & \sum_{\varepsilon_{1}, \ldots, \varepsilon_{n} \in\{0,1\}} \sum_{\gamma \in \mathbb{Z}}\left\|A_{\varepsilon_{n}} \cdots A_{\varepsilon_{1}} v(\gamma)\right\|_{p}^{p}
\end{aligned}
$$

This verifies (4.9) for $1 \leq p<\infty$. For the case $p=\infty$, we have

$$
\left\|a_{n} * v\right\|_{\infty}=\max _{\varepsilon_{1}, \ldots, \varepsilon_{n} \in\{0,1\}} \sup _{\gamma \in \mathbb{Z}}\left\|A_{\varepsilon_{n}} \cdots A_{\varepsilon_{1}} v(\gamma)\right\|_{\infty}=\left\|\mathcal{A}^{n} v\right\|_{\infty}
$$

as desired. Taking $v=y \delta_{\beta}$ in (4.9), we obtain

$$
\left\|\mathcal{A}^{n}\left(y \delta_{\beta}\right)\right\|_{p}=\left\|a_{n}(\cdot-\beta) y\right\|_{p}=\left\|a_{n} y\right\|_{p} .
$$

This establishes the first identity in (4.10). Choosing $v=y \nabla \delta_{\beta}$ in (4.9), we get the second identity in (4.10). 


\section{Characterization of CONVergence}

In this section we give a characterization for the $L_{p}$-convergence $(1 \leq p \leq \infty)$ of the subdivision scheme.

Let $a$ be an element in $\left(\ell_{0}(\mathbb{Z})\right)^{r \times r}$, and $A_{\varepsilon}(\varepsilon \in \mathbb{Z})$ the linear operators on $\left(\ell_{0}(\mathbb{Z})\right)^{r}$ given by $(4.8)$. Let

$$
U:=\left\{v \in\left(\ell_{0}(\mathbb{Z})\right)^{r}: e_{1}^{T} \sum_{\beta \in \mathbb{Z}} v(\beta)=0\right\} .
$$

Lemma 5.1. Let $a$ be an element in $\left(\ell_{0}(\mathbb{Z})\right)^{r \times r}$ such that the matrix $M=\sum_{\alpha \in \mathbb{Z}} a(\alpha) / 2$ satisfies the conditions in (1.6). Then $U$ is invariant under both $A_{0}$ and $A_{1}$ if and only if

$$
e_{1}^{T} \sum_{\beta \in \mathbb{Z}} a(2 \beta)=e_{1}^{T} \sum_{\beta \in \mathbb{Z}} a(2 \beta+1)=e_{1}^{T} .
$$

Proof. Suppose $a$ satisfies the conditions in (5.2). Let $v \in\left(\ell_{0}(\mathbb{Z})\right)^{r}$. Then for $\varepsilon=0,1$,

$$
\begin{aligned}
e_{1}^{T} \sum_{\alpha \in \mathbb{Z}} A_{\varepsilon} v(\alpha) & =e_{1}^{T} \sum_{\alpha \in \mathbb{Z}} \sum_{\beta \in \mathbb{Z}} a(\varepsilon+2 \alpha-\beta) v(\beta) \\
& =\sum_{\beta \in \mathbb{Z}}\left[e_{1}^{T} \sum_{\alpha \in \mathbb{Z}} a(\varepsilon+2 \alpha-\beta)\right] v(\beta)=\sum_{\beta \in \mathbb{Z}} e_{1}^{T} v(\beta) .
\end{aligned}
$$

Hence $v \in U$ implies $A_{\varepsilon} v \in U$. This shows that $U$ is invariant under both $A_{0}$ and $A_{1}$.

Conversely, suppose $U$ is invariant under $A_{0}$. Since $e_{j} \nabla \delta \in U$, we have $A_{0}\left(e_{j} \nabla \delta\right) \in U$ for $j=1, \ldots, r$. Hence

$$
e_{1}^{T} \sum_{\alpha \in \mathbb{Z}}[a(2 \alpha)-a(2 \alpha-1)] e_{j}=e_{1}^{T} \sum_{\alpha \in \mathbb{Z}} A_{0}\left(e_{j} \nabla \delta\right)(\alpha)=0, \quad j=1, \ldots, r .
$$

It follows that

$$
e_{1}^{T} \sum_{\alpha \in \mathbb{Z}} a(2 \alpha)=e_{1}^{T} \sum_{\alpha \in \mathbb{Z}} a(2 \alpha-1)
$$

But (1.6) implies

$$
e_{1}^{T} \sum_{\alpha \in \mathbb{Z}}[a(2 \alpha)+a(2 \alpha-1)]=2 e_{1}^{T} .
$$

The above two relations yield the desired result (5.2).

Lemma 5.2. Suppose $a$ is an element in $\left(\ell_{0}(\mathbb{Z})\right)^{r \times r}$ satisfying (5.2). Let $W$ be the common invariant subspace of $A_{0}$ and $A_{1}$ generated by $e_{1} \nabla \delta, e_{2} \delta, \ldots, e_{r} \delta$, and let $V \subset U$ be a finite dimensional invariant subspace of $A_{0}$ and $A_{1}$ containing $W$. Then

$$
\rho_{p}\left(\left.A_{0}\right|_{W},\left.A_{1}\right|_{W}\right)=\rho_{p}\left(\left.A_{0}\right|_{V},\left.A_{1}\right|_{V}\right), \quad 1 \leq p \leq \infty .
$$

Proof. Obviously, $\rho_{p}\left(\left.A_{0}\right|_{W},\left.A_{1}\right|_{W}\right) \leq \rho_{p}\left(\left.A_{0}\right|_{V},\left.A_{1}\right|_{V}\right)$. In order to prove the reverse inequality, we observe that an element $v$ of $V$ is a finite linear combination of the vectors of the form $e_{1} \nabla \delta_{\beta}, e_{2} \delta_{\beta}, \ldots, e_{r} \delta_{\beta}$, where $\beta \in \mathbb{Z}$. By (4.10) we have

$$
\left\|\mathcal{A}^{n}\left(e_{j} \nabla \delta_{\beta}\right)\right\|_{p}=\left\|\mathcal{A}^{n}\left(e_{j} \nabla \delta\right)\right\|_{p} \quad \text { and } \quad\left\|\mathcal{A}^{n}\left(e_{j} \delta_{\beta}\right)\right\|_{p}=\left\|\mathcal{A}^{n}\left(e_{j} \delta\right)\right\|_{p}
$$


for $j=1, \ldots, r, 1 \leq p \leq \infty$, and $n=1,2, \ldots$ It follows that

$$
\begin{aligned}
\lim _{n \rightarrow \infty}\left\|\mathcal{A}^{n} v\right\|_{p}^{1 / n} & \leq \lim _{n \rightarrow \infty} \max \left\{\left\|\mathcal{A}^{n}\left(e_{1} \nabla \delta\right)\right\|_{p}^{1 / n}, \max _{j=2, \ldots, r}\left\{\left\|\mathcal{A}^{n}\left(e_{j} \delta\right)\right\|_{p}^{1 / n}\right\}\right\} \\
& =\rho_{p}\left(\left.A_{0}\right|_{W},\left.A_{1}\right|_{W}\right) .
\end{aligned}
$$

This shows $\rho_{p}\left(\left.A_{0}\right|_{V},\left.A_{1}\right|_{V}\right) \leq \rho_{p}\left(\left.A_{0}\right|_{W},\left.A_{1}\right|_{W}\right)$, as desired.

Suppose $a$ is supported on $[0, N]$, where $N$ is an integer greater than 1 . If $a$ is supported on $[0,1]$, we set $N=2$. Let $V:=(\ell([0, N-1]))^{r} \cap U$, where $U$ is given by $(5.1)$.

Theorem 5.3. Let $a$ be an element in $\left(\ell_{0}(\mathbb{Z})\right)^{r \times r}$ such that the matrix $M=$ $\sum_{\alpha \in \mathbb{Z}} a(\alpha) / 2$ satisfies the conditions in (1.6). Then the subdivision scheme associated with a converges in the $L_{p}$-norm $(1 \leq p \leq \infty)$ if and only if the following two conditions are satisfied:

(a) $V$ is invariant under $A_{0}$ and $A_{1}$;

(b) $\rho_{p}\left(\left.A_{0}\right|_{V},\left.A_{1}\right|_{V}\right)<2^{1 / p}$.

Proof. Let us first prove that condition (a) is necessary for the $L_{p}$-convergence of the subdivision scheme associated with $a$. By Theorem 2.1, the condition (5.2) is necessary for the subdivision scheme to converge in the $L_{p}$-norm. Hence $U$ is invariant under $A_{0}$ and $A_{1}$, by Lemma 5.1. Moreover, $(\ell([0, N-1]))^{r}$ is invariant under $A_{0}$ and $A_{1}$. Therefore, $V$ is invariant under $A_{0}$ and $A_{1}$.

In order to prove that condition (b) is necessary, we choose $f=e_{j} \chi$, where $1 \leq j \leq r$ and $\chi$ is the characteristic function of the unit interval $[0,1)$. Then by (4.1) we have

$$
Q_{a}^{n} f=\sum_{\alpha \in \mathbb{Z}} a_{n}(\alpha) f\left(2^{n} \cdot-\alpha\right)=\sum_{\alpha \in \mathbb{Z}}\left[a_{n}(\alpha) e_{j}\right] \chi\left(2^{n} \cdot-\alpha\right),
$$

where $a_{n}(n=1,2, \ldots)$ are given by the iteration relation (4.2). Since $\chi$ is the characteristic function of $[0,1)$, we have

$$
\left\|Q_{a}^{n} f\right\|_{p}=2^{-n / p}\left\|a_{n} e_{j}\right\|_{p} .
$$

By Lemma 4.3, it follows that

$$
2^{-n / p}\left\|\mathcal{A}^{n}\left(e_{j} \delta_{\beta}\right)\right\|_{p}=2^{-n / p}\left\|a_{n} e_{j}\right\|_{p}=\left\|Q_{a}^{n} f\right\|_{p} \quad \forall \beta \in \mathbb{Z} .
$$

For $2 \leq j \leq r$, both $e_{1} \chi$ and $e_{1} \chi+e_{j} \chi$ satisfy the moment conditions of order 1 ; hence both $Q_{a}^{n}\left(e_{1} \chi\right)$ and $Q_{a}^{n}\left(e_{1} \chi+e_{j} \chi\right)$ converge to the normalized solution $\phi$ of the refinement equation (1.1) in the $L_{p}$-norm. This shows that $\left\|Q_{a}^{n}\left(e_{j} \chi\right)\right\|_{p} \rightarrow 0$ as $n \rightarrow \infty$. Hence

$$
\lim _{n \rightarrow \infty} 2^{-n / p}\left\|\mathcal{A}^{n}\left(e_{j} \delta_{\beta}\right)\right\|_{p}=0 \quad \forall \beta \in \mathbb{Z} \text { and } j=2, \ldots, r .
$$

Similarly, for $f=e_{1} \chi$, we have

$$
Q_{a}^{n} f-Q_{a}^{n} f\left(\cdot-1 / 2^{n}\right)=\sum_{\alpha \in \mathbb{Z}} \nabla a_{n}(\alpha) f\left(2^{n} \cdot-\alpha\right)=\sum_{\alpha \in \mathbb{Z}}\left[\nabla a_{n}(\alpha) e_{1}\right] \chi\left(2^{n} \cdot-\alpha\right) .
$$

Consequently,

$$
2^{-n / p}\left\|\nabla a_{n} e_{1}\right\|_{p}=\left\|Q_{a}^{n} f-Q_{a}^{n} f\left(\cdot-1 / 2^{n}\right)\right\|_{p}
$$


But

$$
\begin{aligned}
& \left\|Q_{a}^{n} f-Q_{a}^{n} f\left(\cdot-1 / 2^{n}\right)\right\|_{p} \\
& \leq\left\|Q_{a}^{n} f-\phi\right\|_{p}+\left\|\phi-\phi\left(\cdot-1 / 2^{n}\right)\right\|_{p}+\left\|Q_{a}^{n} f\left(\cdot-1 / 2^{n}\right)-\phi\left(\cdot-1 / 2^{n}\right)\right\|_{p} .
\end{aligned}
$$

Hence $\left\|Q_{a}^{n} f-Q_{a}^{n} f\left(\cdot-1 / 2^{n}\right)\right\|_{p} \rightarrow 0$ as $n \rightarrow \infty$. By Lemma 4.3 , it follows that

$$
\lim _{n \rightarrow \infty} 2^{-n / p}\left\|\mathcal{A}^{n}\left(e_{1} \nabla \delta_{\beta}\right)\right\|_{p}=\lim _{n \rightarrow \infty} 2^{-n / p}\left\|\nabla a_{n} e_{1}\right\|_{p}=0 \quad \forall \beta \in \mathbb{Z} .
$$

Therefore (5.3) and (5.4) tell us that

$$
\lim _{n \rightarrow \infty} 2^{-n / p}\left\|\left.\mathcal{A}^{n}\right|_{V}\right\|_{p}=0
$$

Consequently,

$$
2^{-1 / p} \rho_{p}\left(\left.A_{0}\right|_{V},\left.A_{1}\right|_{V}\right)=\lim _{n \rightarrow \infty} 2^{-1 / p}\left\|\left.\mathcal{A}^{n}\right|_{V}\right\|_{p}^{1 / n}<1 .
$$

This completes the proof for the necessity part.

In order to prove the sufficiency part of the theorem, we first observe from the proof of Lemma 5.1 that the condition (5.2) follows from (1.6) and condition (a).

Next, choose $f^{1}:=\varphi$ and $f^{j}:=\varphi(2 r \cdot-2(j-1))$ for $j=2, \ldots, r$, where $\varphi$ is the hat function supported on $[0,2]$ satisfying $\varphi(x)=x$ for $0 \leq x \leq 1$ and $\varphi(x)=2-x$ for $1<x \leq 2$. Then the shifts of $f^{1}, \ldots, f^{r}$ are stable and the vector $f:=\left(f^{1}, \ldots, f^{r}\right)^{T}$ satisfies the moment conditions of order 1 . Thus, by Theorem 3.1 , in order to prove that the subdivision scheme associated with $a$ converges in the $L_{p}$-norm, it suffices to show that $Q_{a}^{n} f$ converges in the $L_{p}$-norm.

Let $g_{j}:=e_{j} f^{j}$ for $j=1, \ldots, r$. Then $f=g_{1}+\cdots+g_{r}$. Thus, it suffices to show that $Q_{a}^{n} g_{j}$ converges in the $L_{p}$-norm for each $j=1, \ldots, r$. This requires the following consequence of condition (b): For $\rho:=\rho_{p}\left(\left.A_{0}\right|_{V},\left.A_{1}\right|_{V}\right)$, any $v \in V$, and any number $\sigma$ in the range $2^{-1 / p} \rho<\sigma<1$, there is a constant $C$ independent of $n$ such that

$$
2^{-n / p}\left\|\mathcal{A}^{n} v\right\|_{p} \leq C \sigma^{n}
$$

For $j=2, \ldots, r$, we have

$$
Q_{a}^{n} g_{j}=\sum_{\alpha \in \mathbb{Z}}\left[a_{n}(\alpha) e_{j}\right] f^{j}\left(2^{n} \cdot-\alpha\right) .
$$

By the choice of $f^{j}$ we obtain

$$
\left\|Q_{a}^{n} g_{j}\right\|_{p} \leq 2^{1-n / p}\left\|a_{n} e_{j}\right\|_{p}=2^{1-n / p}\left\|\mathcal{A}^{n}\left(e_{j} \delta\right)\right\|_{p},
$$

the last equality by Lemma 4.3. The right-hand side of this inequality converges to zero by (5.5) since $e_{j} \delta \in V$. Consequently,

$$
\lim _{n \rightarrow \infty}\left\|Q_{a}^{n} g_{j}\right\|_{p}=0, \quad j=2, \ldots, r .
$$

It remains to deal with the case $j=1$. Since $\varphi=\varphi(2 \cdot) / 2+\varphi(2 \cdot-1)+\varphi(2 \cdot-2) / 2$, we have

$$
\begin{gathered}
Q_{a}^{n} g_{1}=\sum_{\alpha \in \mathbb{Z}} \frac{1}{2}\left[a_{n}(\alpha) e_{1}+a_{n}(\alpha-1) e_{1}\right] \varphi\left(2^{n+1} \cdot-2 \alpha\right) \\
+\sum_{\alpha \in \mathbb{Z}}\left[a_{n}(\alpha) e_{1}\right] \varphi\left(2^{n+1} \cdot-2 \alpha-1\right) .
\end{gathered}
$$


Moreover,

$$
\begin{aligned}
Q_{a}^{n+1} g_{1}=\sum_{\alpha \in \mathbb{Z}} & {\left[a_{n+1}(2 \alpha) e_{1}\right] \varphi\left(2^{n+1} \cdot-2 \alpha\right) } \\
& +\sum_{\alpha \in \mathbb{Z}}\left[a_{n+1}(2 \alpha+1) e_{1}\right] \varphi\left(2^{n+1} \cdot-2 \alpha-1\right) .
\end{aligned}
$$

Subtracting the first from the second, we obtain

$$
Q_{a}^{n+1} g_{1}-Q_{a}^{n} g_{1}=\sum_{\alpha \in \mathbb{Z}}\left[b_{n}(\alpha) e_{1}\right] \varphi\left(2^{n+1} \cdot-2 \alpha\right)+\sum_{\alpha \in \mathbb{Z}}\left[c_{n}(\alpha) e_{1}\right] \varphi\left(2^{n+1} \cdot-2 \alpha-1\right),
$$

where

$$
\begin{aligned}
& b_{n}(\alpha):=a_{n+1}(2 \alpha)-\frac{1}{2} a_{n}(\alpha)-\frac{1}{2} a_{n}(\alpha-1) \quad \text { and } \\
& c_{n}(\alpha):=a_{n+1}(2 \alpha+1)-a_{n}(\alpha), \quad \alpha \in \mathbb{Z} .
\end{aligned}
$$

It follows that

$$
\left\|Q_{a}^{n+1} g_{1}-Q_{a}^{n} g_{1}\right\|_{p} \leq 2^{1-(n+1) / p}\left(\left\|b_{n} e_{1}\right\|_{p}+\left\|c_{n} e_{1}\right\|_{p}\right) .
$$

Let us estimate $\left\|b_{n} e_{1}\right\|_{p}$. Suppose $\alpha=\varepsilon_{1}+2 \varepsilon_{2}+\cdots+2^{n-1} \varepsilon_{n}+2^{n} \gamma$, where $\gamma \in \mathbb{Z}$ and $\varepsilon_{1}, \ldots, \varepsilon_{n} \in\{0,1\}$. Then by Lemma 4.1 we have

$$
b_{n}(\alpha) e_{1}=A_{\varepsilon_{n}} \cdots A_{\varepsilon_{1}} v(\gamma)
$$

where

$$
v:=A_{0}\left(e_{1} \delta\right)-\left[e_{1} \delta+e_{1} \delta_{1}\right] / 2
$$

Since $a$ satisfies (5.2), it follows from the proof of Lemma 5.1 that

$$
\sum_{\beta \in \mathbb{Z}} e_{1}^{T}\left(A_{0}\left(e_{1} \delta\right)\right)(\beta)=\sum_{\beta \in \mathbb{Z}} e_{1}^{T} e_{1} \delta(\beta)=1 .
$$

Hence $\sum_{\beta \in \mathbb{Z}} e_{1}^{T} v(\beta)=0$. This shows $v \in V$. By (5.5) we conclude that there exists a constant $C>0$ such that

$$
2^{-n / p}\left\|b_{n} e_{1}\right\|_{p} \leq C \sigma^{n} \quad \forall n \in \mathbb{N} .
$$

Similarly, there exists a constant $C>0$ such that

$$
2^{-n / p}\left\|c_{n} e_{1}\right\|_{p} \leq C \sigma^{n} \quad \forall n \in \mathbb{N} .
$$

Therefore we obtain

$$
\left\|Q_{a}^{n+1} g_{1}-Q_{a}^{n} g_{1}\right\|_{p} \leq 2^{1-(n+1) / p}\left(\left\|b_{n} e_{1}\right\|_{p}+\left\|c_{n} e_{1}\right\|_{p}\right) \leq 4 C \sigma^{n} \quad \forall n \in \mathbb{N} .
$$

Since $\sigma<1$, this shows that $Q_{a}^{n} g_{1}$ converges in the $L_{p}$-norm. The proof of the theorem is complete.

In the scalar case $(r=1)$, uniform convergence of subdivision schemes was considered by Micchelli and Prautzsch [27], by Daubechies and Lagarias [6], and by Dyn, Gregory, and Levin [8]. In particular, Daubechies and Lagarias used the uniform joint spectral radius in their study of regularity of refinable functions. Employing the $p$-norm joint spectral radius, Jia gave a characterization for $L_{p^{-}}$ convergence in [16].

In the vector case $(r>1)$, using the factorization technique proposed by Plonka [28], Cohen, Daubechies, and Plonka in [3] gave some sufficient conditions for $L_{\infty^{-}}$ convergence and $L_{2}$-convergence of the cascade algorithm. For the case $p=\infty$, 
Cohen, Dyn, and Levin [4] discussed matrix subdivision schemes under a condition weaker than (1.6). Recently, Zhou [34] investigated existence of the solutions of the refinement equation (1.2) without assuming the conditions in (1.6).

In the multidimensional case, convergence of subdivision schemes was studied by Han and Jia [11] for the general $L_{p}$-norm $(1 \leq p \leq \infty)$, and by Lawton, Lee, and Shen [24] for the $L_{2}$-norm.

\section{EXAMPLES}

In this section we give examples in the case $r=2$ to illustrate the general theory.

We want to find real-valued double refinable functions $\phi=\left(\phi_{1}, \phi_{2}\right)^{T}$ as solutions to $L_{p}$-convergent subdivision schemes with matrix masks supported on $[0, N], N=$ 1,2. All the parameters in this section are assumed to be real-valued. We have the basic restrictions from (1.6)

$$
\sum_{j=0}^{N} a(j) / 2=\left[\begin{array}{ll}
1 & 0 \\
0 & \Gamma
\end{array}\right]
$$

with $|\Gamma|<1$, while from Theorem 2.1 we require

$$
e_{1}^{T}(a(0)+a(2))=e_{1}^{T} a(1)=e_{1}^{T} .
$$

To reduce the number of possibilities further, we require that the solution $\phi$ have central symmetries on the support $[0, N]$. Specifically, in that case we will assume that $\phi_{1}$ is centrally symmetric and $\phi_{2}$ is centrally anti-symmetric. Then

$$
\phi=\left[\begin{array}{l}
\phi_{1} \\
\phi_{2}
\end{array}\right] \quad \text { and } P:=\left[\begin{array}{cc}
1 & 0 \\
0 & -1
\end{array}\right] \quad \Longrightarrow \quad \phi=P \phi(N-\cdot) .
$$

Using this in the refinement equation (1.2), we find that

$$
\begin{aligned}
\phi & =P \phi(N-\cdot)=\sum_{\alpha \in \mathbb{Z}} P a(\alpha) \phi(2 N-2 \cdot-\alpha) \\
& =\sum_{\alpha \in \mathbb{Z}} P a(\alpha) P \phi(2 \cdot+\alpha-N)=\sum_{\alpha \in \mathbb{Z}} \operatorname{Pa}(N-\alpha) P \phi(2 \cdot-\alpha) .
\end{aligned}
$$

Thus, $\phi$ will have the desired symmetries, provided

$$
P a(N-\alpha) P=a(\alpha) \quad \forall \alpha \in \mathbb{Z} .
$$

$\mathbf{N}=$ 1. From the requirements (6.1) and (6.2), the mask has the form

$$
a(0):=\left[\begin{array}{ll}
1 & 0 \\
t & y
\end{array}\right], \quad \text { and } \quad a(1):=\left[\begin{array}{cc}
1 & 0 \\
-t & z
\end{array}\right]
$$

with the restriction $|y+z|<2$. We choose the basis $\left\{e_{1} \nabla \delta, e_{2} \delta, e_{2} \delta_{1}\right\}$ for the subspace $V:=\left\{v \in(\ell([0,1]))^{2}: e_{1}^{T} \sum_{\beta \in \mathbb{Z}} v(\beta)=0\right\}$. With respect to this basis, the linear operators $A_{0}$ and $A_{1}$ have the following matrix representations:

$$
\left.A_{0}\right|_{V}=\left[\begin{array}{ccc}
1 & t & t \\
0 & y & 0 \\
0 & 0 & z
\end{array}\right] \text { and }\left.A_{1}\right|_{V}=\left[\begin{array}{ccc}
0 & -2 t & 0 \\
0 & z & 0 \\
0 & y & 0
\end{array}\right] \text {. }
$$

By Lemma 4.2 applied twice,

$$
\rho_{p}\left(\left.A_{0}\right|_{V},\left.A_{1}\right|_{V}\right)=\max \{1, \rho,|z|\},
$$


where $\rho$ is the $p$-norm joint spectral radius of the two $1 \times 1$ matrices $B_{0}=(y)$ and $B_{1}=(z)$.

Let $\mathcal{B}=\left\{B_{0}, B_{1}\right\}$. For $\varepsilon_{1}, \ldots, \varepsilon_{n} \in\{0,1\}$ we have

$$
B_{\varepsilon_{1}} \cdots B_{\varepsilon_{n}}=\prod_{j=1}^{n}\left(y^{1-\varepsilon_{j}} z^{\varepsilon_{j}}\right) \text {. }
$$

Hence for $1 \leq p<\infty$,

$$
\left\|\mathcal{B}^{n}\right\|_{p}^{p}=\left(|y|^{p}+|z|^{p}\right)^{n}
$$

while

$$
\left\|\mathcal{B}^{n}\right\|_{\infty}=(\max \{|y|,|z|\})^{n} .
$$

Therefore we obtain the exact formula for the joint spectral radius:

$$
\rho_{p}\left(\left.A_{0}\right|_{V},\left.A_{1}\right|_{V}\right)= \begin{cases}\max \left\{1,\left(|y|^{p}+|z|^{p}\right)^{1 / p}\right\} & \text { if } 1 \leq p<\infty, \\ \max \{1,|y|,|z|\} & \text { if } p=\infty\end{cases}
$$

All of the discussion above leads to the following example.

Example 6.1. For $1 \leq p<\infty$, the subdivision scheme associated with the mask $a$ supported in $[0,1]$ given by (6.4) converges in the $L_{p}$-norm to the function $\phi:=$ $\left(\phi_{1}, \phi_{2}\right)^{T}$ if and only if $|y|^{p}+|z|^{p}<2$. The subdivision scheme associated with this mask never converges in the $L_{\infty}$-norm. Moreover, if we require the condition (6.3) for the central symmetry of the solution, then $y=z$. In this case, the condition reduces to $|y|<1$ for any $p$, so that convergence holds in any $L_{p}$-norm with $1 \leq p<\infty$.

$\mathbf{N}=\mathbf{2}$. In this case, the requirements (6.1), (6.2), and (6.3) mean that the mask has the form

$$
a(0)=\left[\begin{array}{cc}
\frac{1}{2} & \frac{s}{2} \\
t & \lambda
\end{array}\right], \quad a(1)=\left[\begin{array}{cc}
1 & 0 \\
0 & \mu
\end{array}\right], \quad \text { and } \quad a(2)=\left[\begin{array}{cc}
\frac{1}{2} & -\frac{s}{2} \\
-t & \lambda
\end{array}\right]
$$

where

$$
|2 \lambda+\mu|<2
$$

For the subspace

$$
V:=\left\{v \in(\ell([0,1]))^{2}: e_{1}^{T} \sum_{\beta \in \mathbb{Z}} v(\beta)=0\right\},
$$

we take the basis

$$
v_{1}:=m e_{1} \nabla \delta, \quad v_{2}:=e_{2} \delta, \quad \text { and } \quad v_{3}:=e_{2} \delta_{1},
$$

where $m \neq 0$ is a real number to be chosen. Then the matrices $A_{0}$ and $A_{1}$ restricted to $V$ under this basis become

$$
\left.A_{0}\right|_{V}:=\left[\begin{array}{ccc}
\frac{1}{2} & m t & -m t \\
\frac{s}{2 m} & \lambda & \lambda \\
0 & 0 & \mu
\end{array}\right] \text { and }\left.A_{1}\right|_{V}:=\left[\begin{array}{ccc}
\frac{1}{2} & -m t & m t \\
0 & \mu & 0 \\
\frac{s}{2 m} & \lambda & \lambda
\end{array}\right] .
$$

We observe that the uniform joint spectral radius of the two matrices

$$
\left[\begin{array}{cc}
\lambda & \lambda \\
0 & 0
\end{array}\right] \quad \text { and } \quad\left[\begin{array}{cc}
0 & 0 \\
\lambda & \lambda
\end{array}\right]
$$


is $|\lambda|$. Thus, if $s t=0$ and $\mu=0$, then by Lemma 4.2 we find

$$
\rho_{\infty}\left(\left.A_{0}\right|_{V},\left.A_{1}\right|_{V}\right)=\max \{1 / 2,|\lambda|\} .
$$

If $s t=0$ and $\mu \neq 0$, then $\left.A_{0}\right|_{V}$ and $\left.A_{1}\right|_{V}$ are block triangular, and by Lemma 4.2 we obtain

$$
\rho_{\infty}\left(\left.A_{0}\right|_{V},\left.A_{1}\right|_{V}\right)=\max \left\{1 / 2,|\mu| \rho_{\infty}\left(B_{0}, B_{1}\right)\right\},
$$

where

$$
B_{0}:=\left[\begin{array}{ll}
c & c \\
0 & 1
\end{array}\right] \quad \text { and } \quad B_{1}:=\left[\begin{array}{ll}
1 & 0 \\
c & c
\end{array}\right] \quad \text { with } c=\frac{\lambda}{\mu} .
$$

The uniform joint spectral radius of the two matrices $B_{0}$ and $B_{1}$ was calculated explicitly in [10, Proposition 3.2] and [31, Example 9.2]:

$$
\rho_{\infty}\left(B_{0}, B_{1}\right)= \begin{cases}1 & \text { if }-1 \leq c \leq 1 / 2, \\ \left(c+\sqrt{c^{2}+4 c}\right) / 2 & \text { if } c>1 / 2, \text { and } \\ |c| & \text { if } c<-1 .\end{cases}
$$

This in connection with (6.9) yields

$$
\rho_{\infty}\left(\left.A_{0}\right|_{V},\left.A_{1}\right|_{V}\right)= \begin{cases}\max \{1 / 2,|\mu|\} & \text { if }-1 \leq \lambda / \mu \leq 1 / 2, \\ \max \{1 / 2,|\lambda|\} & \text { if } \lambda / \mu<-1, \text { and } \\ \max \left\{1,|\lambda|+\sqrt{\lambda^{2}+4|\lambda \mu|}\right\} / 2 & \text { if } 1 / 2<\lambda / \mu\end{cases}
$$

For the case $s t \neq 0$, we remark that

$$
\rho_{\infty}\left(\left.A_{0}\right|_{V},\left.A_{1}\right|_{V}\right) \leq \max \left\{\left\|A_{0}\right\|,\left\|A_{1}\right\|\right\}
$$

holds true for any matrix norm $\|\cdot\|$. Using different norms to find an upper bound for the joint spectral radius via (6.11) makes the estimate depend on the choice of basis used in the representation of $\left.A_{\varepsilon}\right|_{V}$. However, the simple choice above does provide sufficient freedom to cover some cases previously discussed in the literature.

Example 6.2. Let $a$ be the element in $\left(\ell_{0}(\mathbb{Z})\right)^{2 \times 2}$ supported in $[0,2]$ given by $(6.5)$.

(a) If $s t=0$, then the subdivision scheme associated with the mask $a$ converges in the $L_{\infty}$-norm if and only if

$$
\max \{|\lambda|,|\mu|\}<1, \quad \lambda(\mu+1)<1, \quad \text { and } \quad \lambda(\mu-1)<1 .
$$

(b) If $s t \neq 0$, then the subdivision scheme associated with the mask $a$ converges in the $L_{\infty}$-norm if $|s t|<1 / 4,|\lambda|<1 / 4$, and $|\mu|<1$.

Consequently, the normalized solution $\phi=\left(\phi_{1}, \phi_{2}\right)^{T}$ of the refinement equation is continuous in each of these two cases.

Proof. By Theorem 5.3, part (a) follows by (6.8) and (6.10).

For part (b), we take $m=s$, in which case

$$
\left\|\left.A_{0}\right|_{V}\right\|_{\infty}=\left\|\left.A_{1}\right|_{V}\right\|_{\infty}=\max \left(\frac{1}{2}+2|s t|, \frac{1}{2}+2|\lambda|,|\mu|\right),
$$

and the result follows by (6.11).

The special case when $s=3 / 2, t=-1 / 8, \lambda=-1 / 8$, and $\mu=1 / 2$ giving the mask

$$
a(0)=\left[\begin{array}{cc}
1 / 2 & 3 / 4 \\
-1 / 8 & -1 / 8
\end{array}\right], \quad a(1)=\left[\begin{array}{cc}
1 & 0 \\
0 & 1 / 2
\end{array}\right], \quad a(2)=\left[\begin{array}{cc}
1 / 2 & -3 / 4 \\
1 / 8 & -1 / 8
\end{array}\right],
$$


appeared in $[13, \S 6]$. The above discussion tells us that the normalized solution $\phi$ of the corresponding refinement equation is continuous.

Let $\phi=\left(\phi^{1}, \ldots, \phi^{r}\right)^{T}$ be a vector of compactly supported distributions on $\mathbb{R}$. We denote by $\mathbb{S}(\phi)$ the shift-invariant space generated by $\phi^{1}, \ldots, \phi^{r}$, that is,

$$
\mathbb{S}(\phi):=\left\{\sum_{j=1}^{r} \sum_{\alpha \in \mathbb{Z}} b_{j}(\alpha) \phi^{j}(\cdot-\alpha): b_{1}, \ldots, b_{r} \in \ell(\mathbb{Z})\right\} .
$$

If $\mathbb{S}(\phi)$ contains all polynomials of degree less than $k$, then we say that $\phi$ has accuracy $k$ (see [13]). If $\phi^{1}, \ldots, \phi^{r}$ belong to $L_{p}(\mathbb{R})(1 \leq p \leq \infty)$, then $\mathbb{S}(\phi)$ provides $L_{p}$-approximation order $k$ if and only if $\phi$ has accuracy $k$ (see [17]). In [19] we provided a characterization for the accuracy of a vector of multiple refinable functions in terms of the corresponding mask.

Let $a$ be the element in $\left(\ell_{0}(\mathbb{Z})\right)^{2 \times 2}$ supported in $[0,2]$ given by $(6.5)$ subject to the condition (6.6). Let $\phi$ be the normalized solution of the corresponding refinement equation. It was proved in [19] that $\phi$ has accuracy 3 if and only if

$$
t \neq 0, \quad \mu=1 / 2, \quad \text { and } \quad \lambda=1 / 4+2 s t .
$$

In this case, the condition (6.6) reduces to

$$
-3 / 4<s t<1 / 4 \text {. }
$$

Example 6.3. Let $a$ be the element in $\left(\ell_{0}(\mathbb{Z})\right)^{2 \times 2}$ supported in $[0,2]$ given by $(6.5)$ subject to the conditions $t \neq 0, \mu=1 / 2, \lambda=1 / 4+2 s t$, and $-3 / 4<s t<1 / 4$. Then the subdivision scheme associated with $a$ converges uniformly. Consequently, the normalized solution $\phi$ of the refinement equation is continuous.

Proof. Let $A_{\varepsilon}(\varepsilon=0,1)$ be the linear operators on $\left(\ell_{0}(\mathbb{Z})\right)^{2}$ as given in (4.8). Suppose $t \neq 0, \mu=1 / 2$, and $\lambda=1 / 4+2 s t$. For the subspace

$$
V:=\left\{v \in(\ell([0,1]))^{2}: e_{1}^{T} \sum_{\beta \in \mathbb{Z}} v(\beta)=0\right\},
$$

we choose the basis

$$
v_{1}=\left[\begin{array}{c}
1 \\
4 t
\end{array}\right] \delta+\left[\begin{array}{c}
-1 \\
4 t
\end{array}\right] \delta_{1}, \quad v_{2}=\left[\begin{array}{l}
0 \\
1
\end{array}\right]\left(\delta-\delta_{1}\right), \quad \text { and } \quad v_{3}=\left[\begin{array}{l}
1 \\
0
\end{array}\right]\left(\delta-\delta_{1}\right)
$$

Then the matrices of $A_{0}$ and $A_{1}$ restricted to $V$ under this basis become

$$
\left.A_{0}\right|_{V}=\left[\begin{array}{ccc}
1 / 2+2 s t & 0 & 0 \\
s / 2 & 1 / 4 & 0 \\
0 & t & 1 / 2
\end{array}\right] \text { and }\left.A_{1}\right|_{V}=\left[\begin{array}{ccc}
1 / 2+2 s t & 0 & 0 \\
-s / 2 & 1 / 4 & 0 \\
0 & -t & 1 / 2
\end{array}\right] \text {. }
$$

Both $\left.A_{0}\right|_{V}$ and $\left.A_{1}\right|_{V}$ are triangular matrices. By Lemma 4.2 we find

$$
\rho_{\infty}\left(\left.A_{0}\right|_{V},\left.A_{1}\right|_{V}\right)=\max \{|1 / 2+2 s t|, 1 / 2\} .
$$

Hence $\rho_{\infty}\left(\left.A_{0}\right|_{V},\left.A_{1}\right|_{V}\right)<1$ if and only if $|1 / 2+2 s t|<1$, i.e., $-3 / 4<s t<1 / 4$. This shows that the subdivision scheme associated with $a$ converges uniformly. Consequently, the normalized solution $\phi$ of the refinement equation is continuous. 


\section{7. $L_{2}$-CONVERGENCE}

In this section we investigate the $L_{2}$-convergence of a subdivision scheme. In the scalar case $(r=1)$, Jia [16] gave a characterization for the $L_{2}$-convergence of a subdivision scheme in terms of the spectral radius of a certain finite matrix associated to the mask. His results were based on the work of Goodman, Micchelli, and Ward [10]. In the vector case $(r>1)$, assuming (2.2), Shen [30] gave a characterization for $L_{2}$-convergence of cascade algorithms. Also see the related work of Long and Mo [26].

The symbol of an element $b \in \ell_{0}(\mathbb{Z})$ is the Laurent polynomial given by

$$
\tilde{b}(z):=\sum_{\alpha \in \mathbb{Z}} b(\alpha) z^{\alpha}, \quad z \in \mathbb{C} \backslash\{0\} .
$$

The symbols of elements in $\left(\ell_{0}(\mathbb{Z})\right)^{r}$ or $\left(\ell_{0}(\mathbb{Z})\right)^{r \times r}$ are defined in a similar way. Suppose $b \in\left(\ell_{0}(\mathbb{Z})\right)^{r \times r}$ and $c \in\left(\ell_{0}(\mathbb{Z})\right)^{r}$. Then the symbol of $b * c$ is given by $\widetilde{b * c}(z)=\tilde{b}(z) \tilde{c}(z)$.

Let $a$ be an element in $\left(\ell_{0}(\mathbb{Z})\right)^{r \times r}$. Define the transition operator $F_{a}$ to be the linear mapping from $\left(\ell_{0}(\mathbb{Z})\right)^{r \times r}$ to $\left(\ell_{0}(\mathbb{Z})\right)^{r \times r}$ given by

$$
F_{a} w(\alpha):=\sum_{\beta, \gamma \in \mathbb{Z}} a(2 \alpha-\beta) w(\beta+\gamma) a(\gamma)^{*} / 2, \quad \alpha \in \mathbb{Z}, w \in\left(\ell_{0}(\mathbb{Z})\right)^{r \times r},
$$

where $a(\gamma)^{*}$ denotes the complex conjugate transpose of $a(\gamma)$. The symbol of $F_{a} w$ has the following form:

$$
\widetilde{F_{a} w}\left(e^{i \xi}\right)=\sum_{j=0,1} \tilde{a}\left((-1)^{j} e^{i \xi / 2}\right) \tilde{w}\left((-1)^{j} e^{i \xi / 2}\right) \tilde{a}\left((-1)^{j} e^{i \xi / 2}\right)^{*} / 4, \quad \xi \in \mathbb{R} .
$$

Indeed, the right-hand side of (7.2) equals

$$
\sum_{\eta, \beta, \gamma \in \mathbb{Z}} a(\eta) w(\beta) a(\gamma)^{*}\left[1+(-1)^{\eta+\beta-\gamma}\right] e^{i(\eta+\beta-\gamma) \xi / 2} / 4 .
$$

Note that $1+(-1)^{\eta+\beta-\gamma}=0$ if $\eta+\beta-\gamma$ is an odd integer, and $1+(-1)^{\eta+\beta-\gamma}=2$ if $\eta+\beta-\gamma=2 \alpha$ for some integer $\alpha$. Therefore, the right-hand side of (7.2) equals

$$
\sum_{\alpha, \beta, \gamma \in \mathbb{Z}} a(2 \alpha-\beta+\gamma) w(\beta) a(\gamma)^{*} e^{i \alpha \xi} / 2,
$$

which is $\widetilde{F_{a} w}\left(e^{i \xi}\right)$. This verifies (7.2).

The form (7.2) of the transition operator $F_{a}$ was introduced by Hervé [14], and the form (7.1) was adopted by Goodman, Jia, and Micchelli [9].

Let us discuss some properties of the transition operator $F_{a}$. Suppose $a$ is supported on $\left[0, N_{a}\right]$, where $N_{a}$ is a positive integer. For a bounded subset $K$ of $\mathbb{R}$, recall that $\ell(K)$ is the linear subspace of $\ell_{0}(\mathbb{Z})$ consisting of all sequences supported on $K \cap \mathbb{Z}$. For a positive integer $N$, let $E_{N}:=(\ell([-N, N]))^{r \times r}$. Then $F_{a}$ maps $E_{N}$ to $E_{\left(N+N_{a}\right) / 2}$ for every integer $N \geq N_{a}$. Suppose $w$ is an eigenvector of $F_{a}$ corresponding to a nonzero eigenvalue $\sigma$. Then the above discussion tells us that $w$ must be supported in $\left[-N_{a}, N_{a}\right]$. This shows that $F_{a}$ has only finitely many nonzero eigenvalues. For an invariant subspace $V$ of $F_{a}$, we define the spectral radius of $\left.F_{a}\right|_{V}$ as

$$
\rho\left(\left.F_{a}\right|_{V}\right):=\rho\left(\left.F_{a}\right|_{V \cap E_{N_{a}}}\right) .
$$

In particular, $\rho\left(F_{a}\right)=\rho\left(\left.F_{a}\right|_{E_{N_{a}}}\right)$. 
We are in a position to state the main result of this section. In the statement of the following theorem, we use $\Delta$ to denote the difference operator on $\ell_{0}(\mathbb{Z})$ given by

$$
\Delta v:=-v(\cdot-1)+2 v-v(\cdot+1), \quad v \in \ell_{0}(\mathbb{Z}) .
$$

In particular, $\Delta \delta:=-\delta_{-1}+2 \delta-\delta_{1}$.

Theorem 7.1. Let $a$ be an element in $\left(\ell_{0}(\mathbb{Z})\right)^{r \times r}$ such that the matrix $M=$ $\sum_{\alpha \in \mathbb{Z}} a(\alpha) / 2$ satisfies the conditions in (1.6). Let $F_{a}$ be the operator given by (7.1). Then the subdivision scheme associated with a converges in the $L_{2}$-norm if and only if

$$
\rho\left(\left.F_{a}\right|_{W}\right)<1
$$

where $W$ is the minimal invariant subspace of $F_{a}$ generated by $e_{1} e_{1}^{T} \Delta \delta, e_{2} e_{2}^{T} \delta, \ldots$, $e_{r} e_{r}^{T} \delta$.

Before proving this theorem, we first establish two auxiliary results.

Lemma 7.2. Suppose $a$ is an element in $\left(\ell_{0}(\mathbb{Z})\right)^{r \times r}$. Let $a_{n}(n=1,2, \ldots)$ be given by the iteration relation (4.2). Then

$$
F_{a}^{n} w(\alpha)=\frac{1}{2^{n+1} \pi} \int_{0}^{2 \pi} \tilde{a}_{n}\left(e^{i \xi}\right) \tilde{w}\left(e^{i \xi}\right) \tilde{a}_{n}\left(e^{i \xi}\right)^{*} e^{-i 2^{n} \alpha \xi} d \xi
$$

holds true for all $w \in\left(\ell_{0}(\mathbb{Z})\right)^{r \times r}$ and $\alpha \in \mathbb{Z}$.

Proof. By the iteration relation (4.2) we have

$$
\tilde{a}_{n}\left(e^{i \xi}\right)=\tilde{a}_{n-1}\left(e^{i 2 \xi}\right) \tilde{a}\left(e^{i \xi}\right), \quad \xi \in \mathbb{R} .
$$

Hence

$$
\begin{aligned}
& \int_{0}^{2 \pi} \tilde{a}_{n}\left(e^{i \xi}\right) \tilde{w}\left(e^{i \xi}\right) \tilde{a}_{n}\left(e^{i \xi}\right)^{*} e^{-i 2^{n} \alpha \xi} d \xi \\
&=\int_{0}^{2 \pi} \tilde{a}_{n-1}\left(e^{i 2 \xi}\right) \tilde{a}\left(e^{i \xi}\right) \tilde{w}\left(e^{i \xi}\right) \tilde{a}\left(e^{i \xi}\right)^{*} \tilde{a}_{n-1}\left(e^{i 2 \xi}\right)^{*} e^{-i 2^{n} \alpha \xi} d \xi \\
&=\frac{1}{2} \int_{0}^{4 \pi} \tilde{a}_{n-1}\left(e^{i \xi}\right) \tilde{a}\left(e^{i \xi / 2}\right) \tilde{w}\left(e^{i \xi / 2}\right) \tilde{a}\left(e^{i \xi / 2}\right)^{*} \tilde{a}_{n-1}\left(e^{i \xi}\right)^{*} e^{-i 2^{n-1} \alpha \xi} d \xi \\
&=\frac{1}{2} \int_{0}^{2 \pi} \tilde{a}_{n-1}\left(e^{i \xi}\right) \tilde{a}\left(e^{i \xi / 2}\right) \tilde{w}\left(e^{i \xi / 2}\right) \tilde{a}\left(e^{i \xi / 2}\right)^{*} \tilde{a}_{n-1}\left(e^{i \xi}\right)^{*} e^{-i 2^{n-1} \alpha \xi} d \xi \\
&+\frac{1}{2} \int_{0}^{2 \pi} \tilde{a}_{n-1}\left(e^{i \xi}\right) \tilde{a}\left(-e^{i \xi / 2}\right) \tilde{w}\left(-e^{i \xi / 2}\right) \tilde{a}\left(-e^{i \xi / 2}\right)^{*} \tilde{a}_{n-1}\left(e^{i \xi}\right)^{*} e^{-i 2^{n-1} \alpha \xi} d \xi \\
&= 2 \int_{0}^{2 \pi} \tilde{a}_{n-1}\left(e^{i \xi}\right) \widetilde{F_{a} w}\left(e^{i \xi}\right) \tilde{a}_{n-1}\left(e^{i \xi}\right)^{*} e^{-i 2^{n-1} \alpha \xi} d \xi .
\end{aligned}
$$

By induction on $n$, we obtain

$$
\begin{aligned}
F_{a}^{n} w(\alpha) & =\frac{1}{2 \pi} \int_{0}^{2 \pi} \widetilde{F_{a}^{n} w}\left(e^{i \xi}\right) e^{-i \alpha \xi} d \xi \\
& =\frac{1}{2^{n+1} \pi} \int_{0}^{2 \pi} \tilde{a}_{n}\left(e^{i \xi}\right) \tilde{w}\left(e^{i \xi}\right) \tilde{a}_{n}\left(e^{i \xi}\right)^{*} e^{-i 2^{n} \alpha \xi} d \xi
\end{aligned}
$$

as desired. 
Lemma 7.3. For any element $v$ of $\left(\ell_{0}(\mathbb{Z})\right)^{r}$,

$$
\lim _{n \rightarrow \infty}\left\|a_{n} * v\right\|_{2}^{1 / n}=\sqrt{2 \rho\left(\left.F_{a}\right|_{W}\right)}
$$

where $W$ is the minimal invariant subspace of $F_{a}$ generated by the element $w \in$ $\left(\ell_{0}(\mathbb{Z})\right)^{r \times r}$ given by

$$
w(\beta):=\sum_{\gamma \in \mathbb{Z}} v(\beta+\gamma) v(\gamma)^{*}, \quad \beta \in \mathbb{Z} .
$$

Proof. Note that $\tilde{w}\left(e^{i \xi}\right)=\tilde{v}\left(e^{i \xi}\right) \tilde{v}\left(e^{i \xi}\right)^{*}, \xi \in \mathbb{R}$. Let $u_{n}:=a_{n} * v, n=1,2, \ldots$ Then $\tilde{u}_{n}\left(e^{i \xi}\right)=\tilde{a}_{n}\left(e^{i \xi}\right) \tilde{v}\left(e^{i \xi}\right), \xi \in \mathbb{R}$. By Parseval's identity, we have

$$
\left\|u_{n}\right\|_{2}^{2}=\sum_{j=1}^{r} e_{j}^{T}\left[\frac{1}{2 \pi} \int_{0}^{2 \pi} \tilde{u}_{n}\left(e^{i \xi}\right) \tilde{u}_{n}\left(e^{i \xi}\right)^{*} d \xi\right] e_{j} .
$$

By using Lemma 7.2 we obtain

$$
\frac{1}{2 \pi} \int_{0}^{2 \pi} \tilde{u}_{n}\left(e^{i \xi}\right) \tilde{u}_{n}\left(e^{i \xi}\right)^{*} d \xi=\frac{1}{2 \pi} \int_{0}^{2 \pi} \tilde{a}_{n}\left(e^{i \xi}\right) \tilde{w}\left(e^{i \xi}\right) \tilde{a}_{n}\left(e^{i \xi}\right)^{*} d \xi=2^{n} F_{a}^{n} w(0) .
$$

Consequently,

$$
\left\|u_{n}\right\|_{2}^{2}=\sum_{j=1}^{r} e_{j}^{T} 2^{n}\left[F_{a}^{n} w(0)\right] e_{j} \leq r 2^{n}\left\|F_{a}^{n} w\right\|_{\infty} .
$$

On the other hand,

$$
\left\|F_{a}^{n} w\right\|_{\infty}=\sup \left\{\left|e_{j}^{T} F_{a}^{n} w(\alpha) e_{k}\right|: \alpha \in \mathbb{Z}, j, k=1, \ldots, r\right\} .
$$

By Lemma 7.2 we have

$$
\begin{aligned}
e_{j}^{T} 2^{n} F_{a}^{n} w(\alpha) e_{k} & =\frac{1}{2 \pi} \int_{0}^{2 \pi} e_{j}^{T} \tilde{a}_{n}\left(e^{i \xi}\right) \tilde{v}\left(e^{i \xi}\right) \tilde{v}\left(e^{i \xi}\right)^{*} \tilde{a}_{n}\left(e^{i \xi}\right)^{*} e_{k} e^{-i 2^{n} \alpha \xi} d \xi \\
& =\frac{1}{2 \pi} \int_{0}^{2 \pi}\left[e_{j}^{T} \tilde{u}_{n}\left(e^{i \xi}\right)\right]\left[e_{k}^{T} \tilde{u}_{n}\left(e^{i \xi}\right)\right]^{*} e^{-i 2^{n} \alpha \xi} d \xi .
\end{aligned}
$$

It follows that

$$
2^{n}\left|e_{j}^{T} F_{a}^{n} w(\alpha) e_{k}\right| \leq\left\|e_{j}^{T} u_{n}\right\|_{2}\left\|e_{k}^{T} u_{n}\right\|_{2} \leq\left\|u_{n}\right\|_{2}^{2} .
$$

This is valid for all $\alpha \in \mathbb{Z}$ and all $j, k=1, \ldots, r$. Therefore, we obtain

$$
2^{n}\left\|F_{a}^{n} w\right\|_{\infty} \leq\left\|u_{n}\right\|_{2}^{2} .
$$

Finally, (7.4) and (7.5) together yield

$$
\lim _{n \rightarrow \infty}\left\|a_{n} * v\right\|_{2}^{1 / n}=\lim _{n \rightarrow \infty}\left[2^{n}\left\|F_{a}^{n} w\right\|_{\infty}\right]^{1 /(2 n)}=\sqrt{2 \rho\left(\left.F_{a}\right|_{W}\right)},
$$

where $W$ is the minimal invariant subspace of $F_{a}$ generated by the element $w$.

Proof of Theorem 7.1. By Theorem 5.3, Lemma 5.2, and Lemma 4.3, the subdivision scheme associated with $a$ converges in the $L_{2}$-norm if and only if

$$
\lim _{n \rightarrow \infty}\left\|a_{n} * v\right\|_{2}^{1 / n}<\sqrt{2}
$$

for $v=e_{1} \nabla \delta, e_{2} \delta, \ldots, e_{r} \delta$. But Lemma 7.3 tells us that

$$
\lim _{n \rightarrow \infty}\left\|a_{n} * v\right\|_{2}^{1 / n}=\lim _{n \rightarrow \infty}\left[2^{n}\left\|F_{a}^{n} w\right\|_{\infty}\right]^{1 /(2 n)},
$$


where $w$ is obtained from $v$ via (7.3). When $v=e_{1} \nabla \delta, e_{2} \delta, \ldots, e_{r} \delta$, the corresponding $w$ will be $e_{1} e_{1}^{T} \Delta \delta, e_{2} e_{2}^{T} \delta, \ldots, e_{r} e_{r}^{T} \delta$, respectively. Let $W$ be the minimal invariant subspace of $F_{a}$ generated by those vectors. We conclude that the subdivision scheme associated with $a$ converges in the $L_{2}$-norm if and only if $\rho\left(\left.F_{a}\right|_{W}\right)<1$.

Now we apply the above results for the refinement masks defined on $[0,2]$ by (6.5) and (6.6) to give a characterization for the convergence in the $L_{2}$-norm.

Let

$$
\begin{aligned}
& w_{1}:=\left[\begin{array}{cc}
-\delta_{-1}+2 \delta-\delta_{1} & 0 \\
0 & 0
\end{array}\right], \quad w_{2}:=\left[\begin{array}{cc}
0 & \delta_{-1}-\delta_{1} \\
\delta_{1}-\delta_{-1} & 0
\end{array}\right], \\
& w_{3}:=\left[\begin{array}{ll}
0 & 0 \\
0 & \delta
\end{array}\right], \quad \text { and } \quad w_{4}:=\left[\begin{array}{cc}
0 & 0 \\
0 & \delta_{-1}+\delta_{1}
\end{array}\right] .
\end{aligned}
$$

By computation we find that

$$
F_{a}\left[\begin{array}{l}
w_{1} \\
w_{2} \\
w_{3} \\
w_{4}
\end{array}\right]=\left[\begin{array}{cccc}
1 / 4 & 0 & 2 t^{2} & -t^{2} \\
s / 2 & \lambda / 2+\mu / 4 & -2 t \mu & t \mu \\
s^{2} / 8 & s \lambda / 4 & \lambda^{2}+\mu^{2} / 2 & \lambda^{2} / 2 \\
0 & s \mu / 4 & 2 \lambda \mu & \lambda \mu
\end{array}\right]\left[\begin{array}{c}
w_{1} \\
w_{2} \\
w_{3} \\
w_{4}
\end{array}\right]
$$

The $4 \times 4$ matrix in (7.6) is denoted by $B$. Let $W$ be the minimal invariant subspace of $F_{a}$ generated by $e_{1} e_{1}^{T} \Delta \delta=w_{1}$ and $e_{2} e_{2}^{T} \delta=w_{3}$.

Example 7.4. Let $a$ be the sequence of $2 \times 2$ matrices given by (6.5) with the parameters satisfying (6.6). The subdivision scheme associated with the mask $a$ converges in the $L_{2}$-norm if and only if $\rho(B)<1$, where $\rho(B)$ denotes the spectral radius of the matrix $B$. In particular, if the normalized solution $\phi$ of the refinement equation with the mask $a$ has accuracy 3 , then the subdivision scheme associated with the mask $a$ converges in the $L_{2}$-norm, provided $|2 \lambda+\mu|<2$.

Proof. By Theorem 7.1, the subdivision scheme associated with the mask $a$ converges in the $L_{2}$-norm if and only if $\rho\left(\left.F_{a}\right|_{W}\right)<1$. We have $\rho\left(\left.F_{a}\right|_{W}\right) \leq \rho(B)$ with equality if $W$ contains $w_{j}$ for all $j=1,2,3,4$. Thus, in order to prove the first statement, it suffices to show that $\rho\left(\left.F_{a}\right|_{W}\right)<1$ implies $\rho(B)<1$.

Consider the case $t \neq 0$ first. In this case, $W$ contains $w_{4}$. If $W$ does not contain $w_{2}$, then we must have $s \lambda=s \mu=0$. Hence

$$
\rho\left(\left.F_{a}\right|_{W}\right)=\max \{1 / 4, \sigma\} \quad \text { and } \quad \rho(B)=\max \{1 / 4,|\lambda / 2+\mu / 4|, \sigma\},
$$

where $\sigma$ is the spectral radius of the $2 \times 2$ matrix

$$
\left[\begin{array}{cc}
\lambda^{2}+\mu^{2} / 2 & \lambda^{2} / 2 \\
2 \lambda \mu & \lambda \mu
\end{array}\right]
$$

It can be easily verified that $\sigma<1$ implies $|\lambda / 2+\mu / 4|<1$. Therefore, $\rho\left(\left.F_{a}\right|_{W}\right)<1$ implies $\rho(B)<1$.

Next, consider the case $t=0$. In this case, if $W$ does not contain $w_{4}$, then we must have $\lambda^{2} / 2=0$. Consequently, $\rho\left(\left.F_{a}\right|_{W}\right)<1$ implies $\mu^{2} / 2<1$. It follows that $\rho(B)<1$. If $W$ contains $w_{4}$, then we have $\rho\left(\left.F_{a}\right|_{W}\right) \geq \sigma$. Again, $\rho\left(\left.F_{a}\right|_{W}\right)<1$ implies $\rho(B)<1$. 
It remains to prove the second statement. If $\phi$ has accuracy 3 , then

$$
t \neq 0, \quad \mu=1 / 2, \quad \text { and } \quad \lambda=1 / 4+2 s t .
$$

In this case, $|2 \lambda+\mu|<2$ is equivalent to $-3 / 4<s t<1 / 4$. Moreover, we find that the eigenvalues of $B$ are $1 / 4,1 / 16,1 / 4+s t$, and $1 / 4+2 s t+4 s^{2} t^{2}$. Thus,

$$
\rho(B)=\max \left\{1 / 4,|1 / 4+s t|,\left|1 / 4+2 s t+4 s^{2} t^{2}\right|\right\} .
$$

Hence $\rho(B)<1$ for $-3 / 4<s t<1 / 4$. Therefore, Theorem 7.1 gives the desired conclusion.

\section{Multiple Wavelets}

In this section we apply the general theory on vector subdivision schemes developed so far to the construction of orthogonal multiple wavelets.

The first nontrivial example of continuous symmetric orthogonal double wavelets was constructed by Donovan, Geronimo, Hardin, and Massopust in [7] by means of fractal interpolation. Here we take the same approach as Chui and Lian did in [2] to the construction of orthogonal double wavelets by using refinement equations.

Our starting point is the following characterization of orthonormality of the shifts of the normalized solution of the refinement equation in terms of the mask. Some different forms of this result were obtained by Long, Chen, and Yuan in [25], and by Shen in [30].

Theorem 8.1. Let $a$ be an element in $\left(\ell_{0}(\mathbb{Z})\right)^{r \times r}$ such that the matrix $M=$ $\sum_{\alpha \in \mathbb{Z}} a(\alpha) / 2$ satisfies (1.6), and let $\phi=\left(\phi^{1}, \cdots, \phi^{r}\right)^{T}$ be the normalized solution of the refinement equation

$$
\phi=\sum_{\alpha \in \mathbb{Z}} a(\alpha) \phi(2 \cdot-\alpha) .
$$

Let

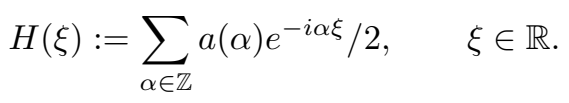

Then $\left\{\phi^{j}(\cdot-\alpha): j=1, \ldots, r, \alpha \in \mathbb{Z}\right\}$ forms an orthonormal system in $L_{2}(\mathbb{R})$ if and only if

(a) $H(\xi) H(\xi)^{*}+H(\xi+\pi) H(\xi+\pi)^{*}=I_{r}$ for all $\xi \in \mathbb{R}$, where $I_{r}$ denotes the $r \times r$ identity matrix, and

(b) the subdivision scheme associated with a converges in the $L_{2}$-norm.

Proof. By Theorem 3.2, condition (b) is necessary. Let us prove that condition (a) is also necessary. It is well known that $\left\{\phi^{j}(\cdot-\alpha): j=1, \ldots, r, \alpha \in \mathbb{Z}\right\}$ forms an orthonormal system in $L_{2}(\mathbb{R})$ if and only if

$$
\sum_{\beta \in \mathbb{Z}} \hat{\phi}(\xi+2 \beta \pi) \hat{\phi}(\xi+2 \beta \pi)^{*}=I_{r} \quad \forall \xi \in \mathbb{R} .
$$

But $\phi$ satisfies the refinement equation with the mask $a$; hence

$$
\hat{\phi}(\xi)=H(\xi / 2) \hat{\phi}(\xi / 2) \quad \forall \xi \in \mathbb{R} .
$$


It follows that, for every $\xi \in \mathbb{R}$,

$$
\begin{aligned}
I_{r}= & \sum_{\beta \in \mathbb{Z}} \hat{\phi}(\xi+2 \beta \pi) \hat{\phi}(\xi+2 \beta \pi)^{*} \\
= & H(\xi / 2)\left[\sum_{\beta \in \mathbb{Z}} \hat{\phi}(\xi / 2+2 \beta \pi) \hat{\phi}(\xi / 2+2 \beta \pi)^{*}\right] H(\xi / 2)^{*} \\
& +H(\xi / 2+\pi)\left[\sum_{\beta \in \mathbb{Z}} \hat{\phi}(\xi / 2+\pi+2 \beta \pi) \hat{\phi}(\xi / 2+\pi+2 \beta \pi)^{*}\right] H(\xi / 2+\pi)^{*} \\
= & H(\xi / 2) H(\xi / 2)^{*}+H(\xi / 2+\pi) H(\xi / 2+\pi)^{*} .
\end{aligned}
$$

This shows that condition (a) is necessary.

Now assume that conditions (a) and (b) are satisfied. Let $f^{1}:=\chi_{[0,1)}$ and, for $j=2, \ldots, r$, let

$$
f^{j}:=\sum_{k=0}^{2^{j}-1}(-1)^{k} \chi_{\left[k / 2^{j},(k+1) / 2^{j}\right)} .
$$

It is easily seen that $\left\{f^{j}(\cdot-\alpha): j=1, \ldots, r, \alpha \in \mathbb{Z}\right\}$ forms an orthonormal system in $L_{2}(\mathbb{R})$. Consequently,

$$
\sum_{\beta \in \mathbb{Z}} \hat{f}(\xi+2 \beta \pi) \hat{f}(\xi+2 \beta \pi)^{*}=I_{r} \quad \forall \xi \in \mathbb{R} .
$$

Since $\widehat{Q_{a} f}(\xi)=\widehat{H}(\xi / 2) \widehat{f}(\xi / 2)$ and condition (a) holds true, by the discussion in the last paragraph and induction we see that

$$
\sum_{\beta \in \mathbb{Z}}\left(Q_{a}^{n} f\right)^{\wedge}(\xi+2 \beta \pi)\left(Q_{a}^{n} f\right)^{\wedge}(\xi+2 \beta \pi)^{*}=I_{r} \quad \forall \xi \in \mathbb{R} .
$$

We observe that $f:=\left(f^{1}, \ldots, f^{r}\right)^{T}$ satisfies the moment conditions of order 1 . By condition (b), $Q_{a}^{n} f$ converges to $\phi$ in the $L_{2}$-norm. Letting $n \rightarrow \infty$ in (8.2), we obtain (8.1), as desired.

It is easily seen that condition (a) of Theorem 8.1 is equivalent to the following condition:

$$
\sum_{\alpha \in \mathbb{Z}} a(\alpha) a(\alpha+2 \gamma)^{*}=2 \delta_{\gamma, 0} I_{r} \quad \forall \gamma \in \mathbb{Z}
$$

Let $\phi=\left(\phi^{1}, \ldots, \phi^{r}\right)^{T}$ be a refinable vector of compactly supported functions in $L_{2}(\mathbb{R})$. For $k \in \mathbb{Z}$, let

$$
V_{k}:=\operatorname{closure}_{L_{2}(\mathbb{R})}\left\{\sum_{\alpha \in \mathbb{Z}} \sum_{j=1}^{r} c_{j}(\alpha) \phi^{j}\left(2^{k} \cdot-\alpha\right): c_{j} \in \ell_{0}(\mathbb{Z})\right\} .
$$

It is known that $\left(V_{k}\right)_{k \in \mathbb{Z}}$ forms a multiresolution of $L_{2}(\mathbb{R})$ (see [21]). Suppose the shifts of $\phi^{1}, \ldots, \phi^{r}$ are orthonormal. Let $W_{k}$ be the orthogonal complement of $V_{k}$ in $V_{k+1}$. Then $L_{2}(\mathbb{R})$ is the orthogonal sum of $W_{k}, k \in \mathbb{Z}$. Functions $\psi^{1}, \ldots, \psi^{r}$ in $W_{0}$ are called orthogonal multiple wavelets if $\left\{\psi^{j}(\cdot-\alpha): j=1, \ldots, r, \alpha \in \mathbb{Z}\right\}$ is an orthonormal basis of $W_{0}$. If this is the case, then

$$
\left\{2^{k / 2} \psi^{j}\left(2^{k} \cdot-\alpha\right): j=1, \ldots, r, k \in \mathbb{Z}, \alpha \in \mathbb{Z}\right\}
$$


is an orthonormal basis of $L_{2}(\mathbb{R})$. Furthermore, if $\psi^{1}, \ldots, \psi^{r}$ are compactly supported, then $\psi=\left(\psi^{1}, \ldots, \psi^{r}\right)^{T}$ can be represented as

$$
\psi=\left(\psi^{1}, \ldots, \psi^{r}\right)^{T}=\sum_{\alpha \in \mathbb{Z}} b(\alpha) \phi(2 \cdot-\alpha),
$$

where $b \in\left(\ell_{0}(\mathbb{Z})\right)^{r}$. The following theorem gives a characterization on $b$ for the orthonormality of the shifts of $\psi^{1}, \ldots, \psi^{r}$.

Theorem 8.2. Let $a$ be an element in $\left(\ell_{0}(\mathbb{Z})\right)^{r \times r}$ such that the matrix $M=$ $\sum_{\alpha \in \mathbb{Z}} a(\alpha) / 2$ satisfies (1.6), and let $\phi=\left(\phi^{1}, \cdots, \phi^{r}\right)^{T}$ be the normalized solution of the refinement equation

$$
\phi=\sum_{\alpha \in \mathbb{Z}} a(\alpha) \phi(2 \cdot-\alpha)
$$

for which $\left\{\phi^{j}(\cdot-\alpha): j=1, \ldots, r, \alpha \in \mathbb{Z}\right\}$ forms an orthonormal system in $L_{2}(\mathbb{R})$. Let $\psi=\left(\psi^{1}, \ldots, \psi^{r}\right)^{T}$ be the vector given in (8.4). Then $\psi^{1}, \ldots, \psi^{r}$ are orthonormal mutliple wavelets for the multiresolution $\left(V_{k}\right)_{k \in \mathbb{Z}}$ if and only if

$$
\sum_{\alpha \in \mathbb{Z}} a(\alpha) b(\alpha+2 \gamma)^{*}=0 \quad \forall \gamma \in \mathbb{Z},
$$

and

$$
\sum_{\alpha \in \mathbb{Z}} b(\alpha) b(\alpha+2 \gamma)^{*}=2 \delta_{\gamma, 0} I_{r} \quad \forall \gamma \in \mathbb{Z} .
$$

Proof. The proof follows standard arguments using the orthonormality of the integer translates of $\phi$, the refinement equation for $\phi$, and the representation (8.4) to show that (8.5) is equivalent to $\psi$ being orthogonal to the $\gamma$ translates of $\phi$ and that (8.6) is equivalent to $\psi$ being orthogonal to the $\gamma$ translates of itself.

Thus, the construction of orthogonal multiple wavelets reduces to a problem of matrix extension. This problem was solved by Lawton, Lee, and Shen in [22]. It will be interesting to know whether it is always possible to construct symmetric multiple wavelets if the multiple refinable functions $\phi^{1}, \ldots, \phi^{r}$ are symmetric.

We continue our examples of the last two sections and investigate the possibility of constructing orthonormal double wavelets with symmetry.

$\mathbf{N}=1$. We use the mask from (6.4). In this case, (8.3) reduces to

$$
a(0) a(0)^{*}+a(1) a(1)^{*}=2 I_{2} .
$$

From Example 6.1, for the mask given by (6.4)

$$
a(0)=\left[\begin{array}{ll}
1 & 0 \\
t & y
\end{array}\right], \quad a(1)=\left[\begin{array}{cc}
1 & 0 \\
-t & z
\end{array}\right],
$$

the associated subdivision scheme converges in the $L_{2}$-norm if and only if $|y|^{2}+$ $|z|^{2}<2$. The orthonormality assumption results in the added restriction

$$
\begin{cases}2 t^{2}+y^{2}+z^{2}=2, & \text { if no symmetry on } \phi \text { is assumed, } \\ t^{2}+y^{2}=1, & \text { if } y=z \text { is assumed to provide the symmetry on } \phi .\end{cases}
$$

In the case when $y=z$ in the mask (6.4), we take

$$
b(0):=\left[\begin{array}{cc}
0 & 1 \\
y & -t
\end{array}\right], \quad \text { and } \quad b(1):=\left[\begin{array}{cc}
0 & -1 \\
-y & -t
\end{array}\right] .
$$


Then (8.5) and (8.6) can be easily verified. With this choice of $b$, the vector $\psi=$ $\left(\psi_{1}, \psi_{2}\right)^{T}$ given in (8.4) induces orthogonal double wavelets $\psi^{1}$ and $\psi^{2}$ such that $\psi^{1}$ is symmetric about $1 / 2$ and $\psi^{2}$ is anti-symmetric about $1 / 2$.

A particular example is provided when $t= \pm 4 / 5$, and $y=z= \pm 3 / 5$. Then $t^{2}+y^{2}=1$ so the shifts of $\phi$ are orthonormal. This special mask is rational. The wavelets obtained for $N=1$ are not continuous.

$\mathbf{N}=\mathbf{2}$. For $N=2$ the equations (8.3) are

$$
a(0) a(2)^{*}=0 \quad \text { and } \quad a(0) a(0)^{*}+a(1) a(1)^{*}+a(2) a(2)^{*}=2 I_{2} .
$$

For the mask (6.5), the first of these equations yields the relations $s^{2}=1, t^{2}=\lambda^{2}$ and $t=s \lambda$, while the second gives the additional requirement that $\mu^{2}=2-4 t^{2}$. We choose $s=1$ and $\mu=\sqrt{2-4 t^{2}}$. Then the mask takes the form

$$
a(0)=\left[\begin{array}{cc}
\frac{1}{2} & \frac{1}{2} \\
t & t
\end{array}\right], \quad a(1)=\left[\begin{array}{cc}
1 & 0 \\
0 & \sqrt{2-4 t^{2}}
\end{array}\right], \quad \text { and } \quad a(2)=\left[\begin{array}{cc}
\frac{1}{2} & -\frac{1}{2} \\
-t & t
\end{array}\right] .
$$

Here the restrictions on the parameters are that $|2 t+\mu|<2$, and, assuming we want a real valued solution, $t^{2} \leq 1 / 2$. From (6.7), we find that the eigenvalues of $\left.A_{0}\right|_{V}$ and $\left.A_{1}\right|_{V}$ are $\sqrt{2-4 t^{2}}, t+1 / 2$, and 0 ; hence

$$
\rho_{\infty}\left(\left.A_{0}\right|_{V},\left.A_{1}\right|_{V}\right) \geq \max \left\{\sqrt{2-4 t^{2}},|t+1 / 2|\right\} .
$$

For convergence in the $L_{\infty}$-norm, $\rho_{\infty}\left(\left.A_{0}\right|_{V},\left.A_{1}\right|_{V}\right)$ must be less than 1 . This further restricts the parameter $t$ to

$$
-1 / \sqrt{2} \leq t<-1 / 2 .
$$

Example 8.3. The subdivision scheme with real-valued mask (8.7) converges uniformly to the normalized solution $\phi=\left(\phi^{1}, \phi^{2}\right)^{T}$ which is supported on [0,2], with $\phi_{1}$ symmetric about 1 and $\phi_{2}$ anti-symmetric about 1 , and for which $\left\{\phi^{j}(\cdot-\alpha)\right.$ : $j=1,2, \alpha \in \mathbb{Z}\}$ forms an orthonormal system in $L_{2}(\mathbb{R})$ for any $t$ satisfying (8.8). Moreover, for the coefficients

$$
b(0):=\left[\begin{array}{cc}
-1 / 2 & -1 / 2 \\
\mu / 2 & \mu / 2
\end{array}\right], \quad b(1):=\left[\begin{array}{cc}
1 & 0 \\
0 & -2 t
\end{array}\right], \quad \text { and } \quad b(2):=\left[\begin{array}{cc}
-1 / 2 & 1 / 2 \\
-\mu / 2 & \mu / 2
\end{array}\right],
$$

where $\mu=\sqrt{2-4 t^{2}}$, the vector

$$
\psi=\left(\psi^{1}, \psi^{2}\right)^{T}:=\sum_{\alpha \in \mathbb{Z}} b(\alpha) \phi(2 \cdot-\alpha)
$$

gives orthogonal double wavelets $\psi^{1}$ and $\psi^{2}$, which are continuous. Moreover, $\psi^{1}$ is symmetric about 1 , and $\psi^{2}$ is anti-symmetric about 1 .

Proof. We first show that the subdivision scheme with mask (8.7) converges in the $L_{\infty}$-norm for any $t$ satisfying (8.8). When we take the basis of $V$ as

$$
\left\{m e_{2} \delta, \quad m e_{2} \delta_{1}, \quad-e_{1} \nabla \delta+e_{2} \delta+e_{2} \delta_{1}\right\},
$$

we have

$$
\left.A_{0}\right|_{V}:=\left[\begin{array}{ccc}
\frac{1}{2}+t & \frac{1}{2}+t & -\frac{m}{2} \\
0 & \sqrt{2-4 t^{2}} & 0 \\
0 & \frac{2 t+\sqrt{2-4 t^{2}}}{m} & 0
\end{array}\right], \quad \text { and }\left.\quad A_{1}\right|_{V}:=\left[\begin{array}{ccc}
\sqrt{2-4 t^{2}} & 0 & 0 \\
\frac{1}{2}+t & \frac{1}{2}+t & -\frac{m}{2} \\
\frac{2 t+\sqrt{2-4 t^{2}}}{m} & 0 & 0
\end{array}\right]
$$


It is not possible to choose an $m$ so that the $\ell_{\infty}$-norms are less than 1 throughout the range $-1 / \sqrt{2} \leq t<-1 / 2$. However, this is nearly possible for the product of any two. For $m=2$, we have

$$
\begin{aligned}
\left.A_{0}\right|_{V} ^{2}= & {\left[\begin{array}{ccc}
\left(\frac{1}{2}+t\right)^{2} & \frac{1}{4}+t \sqrt{2-4 t^{2}}+t^{2} & -\left(\frac{1}{2}+t\right) \\
0 & 2-4 t^{2} & 0 \\
0 & \frac{\sqrt{2-4 t^{2}}\left(\sqrt{2-4 t^{2}}+2 t\right)}{2} & 0
\end{array}\right], } \\
\left.\left.A_{0}\right|_{V} A_{1}\right|_{V}= & {\left[\begin{array}{ccc}
\frac{1}{4}+t \sqrt{2-4 t^{2}}+t^{2} & \left(\frac{1}{2}+t\right)^{2} & -\left(\frac{1}{2}+t\right) \\
\left(\frac{1}{2}+t\right) \sqrt{2-4 t^{2}} & \left(\frac{1}{2}+t\right) \sqrt{2-4 t^{2}} & -\sqrt{2-4 t^{2}} \\
\frac{\left(\frac{1}{2}+t\right)\left(\sqrt{2-4 t^{2}}+2 t\right)}{2} & \frac{\left(\frac{1}{2}+t\right)\left(\sqrt{2-4 t^{2}}+2 t\right)}{2} & -\frac{\left(\sqrt{2-4 t^{2}}+2 t\right)}{2}
\end{array}\right], } \\
\left.A_{1}\right|_{V} ^{2}= & {\left[\begin{array}{ccc}
2-4 t^{2} & 0 & 0 \\
\frac{1}{4}+t \sqrt{2-4 t^{2}}+t^{2} & \left(\frac{1}{2}+t\right)^{2} & -\left(\frac{1}{2}+t\right) \\
\frac{\sqrt{2-4 t^{2}}\left(\sqrt{2-4 t^{2}}+2 t\right)}{2} & 0 & 0
\end{array}\right], } \\
\left.\left.A_{1}\right|_{V} A_{0}\right|_{V}= & {\left[\begin{array}{ccc}
\frac{\left(\frac{1}{2}+t\right) \sqrt{2-4 t^{2}}}{\left(\frac{1}{2}+t\right)^{2}} & \left.\frac{1}{4}+t \sqrt{2}+t\right) \sqrt{2-4 t^{2}}+t^{2} & -\sqrt{2-4 t^{2}} \\
\frac{\left(\frac{1}{2}+t\right)\left(\sqrt{2-4 t^{2}}+2 t\right)}{2} & \frac{\left(\frac{1}{2}+t\right)\left(\sqrt{2-4 t^{2}}+2 t\right)}{2} & -\frac{\left(\sqrt{2-4 t^{2}}+2 t\right)}{2}
\end{array}\right] . }
\end{aligned}
$$

It can be verified directly that the $\ell_{\infty}$-norms of these matrices are less than 1 for the range $-1 / \sqrt{2}<t<-1 / 2$, and consequently, $\left\|\mathcal{A}^{2}\right\|_{\infty}<1$ and therefore $\rho_{\infty}\left(\left.A_{0}\right|_{V},\left.A_{1}\right|_{V}\right)<1$.

When $t=-1 / \sqrt{2}$, we choose $m=31 / 16$ and verify directly that the $\ell_{1}$-norm of both $\left.A_{0}\right|_{V}$ and $\left.A_{1}\right|_{V}$ is $31 / 32$. By (6.11), we again have $\rho_{\infty}\left(\left.A_{0}\right|_{V}, A_{1} \mid V\right)<1$.

When the coefficients $b$ are to be supported on $[0,2]$, the equations (8.5) and (8.6) reduce to

$$
a(0) b(0)^{*}+a(1) b(1)^{*}+a(2) b(2)^{*}=0, \quad a(0) b(2)^{*}=0, \quad a(2) b(0)^{*}=0,
$$

and

$$
b(0) b(0)^{*}+b(1) b(1)^{*}+b(2) b(2)^{*}=2 I_{2}, \quad b(0) b(2)^{*}=0, \quad b(2) b(0)^{*}=0 .
$$

The coefficients (8.9) were chosen specifically to satisfy these relations.

The special choice $t=-\sqrt{7} / 4$ was considered by Chui and Lian in [2]. In this case, the normalized solution $\phi=\left(\phi_{1}, \phi_{2}\right)^{T}$ of the corresponding refinement equation has accuracy 2. In fact, this is the only choice of the parameter $t$ such that $\phi$ has accuracy 2. Chui and Lian did not prove that $\phi^{1}, \phi^{2}$ are functions in $L_{2}(\mathbb{R})$ with orthogonal shifts. We have done that and more by including it in a construction of an entire family of orthogonal double wavelets that are continuous and have symmetry.

\section{REFERENCES}

1. A. S. Cavaretta, W. Dahmen, and C. A. Micchelli, Stationary Subdivision, Memoirs of Amer. Math. Soc., Volume 93, 1991. MR 92h:65017

2. C. K. Chui and J. A. Lian, A study of orthonormal multi-wavelets, J. Applied Numerical Math. 20 (1996), 273-298.CMP 96:16 
3. A. Cohen, I. Daubechies, and G. Plonka, Regularity of refinable function vectors, J. Fourier Anal. Appl. 3 (1997), 295-324. CMP 97:12

4. A. Cohen, N. Dyn, and D. Levin, Stability and inter-dependence of matrix subdivision schemes, in Advanced Topics in Multivariate Approximation, F. Fontanella, K. Jetter and P.-J. Laurent (eds.), 1996, pp. 33-45.

5. W. Dahmen and C. A. Micchelli, Biorthogonal wavelet expansions, Constr. Approx. 13 (1997), 293-328. CMP 97:13

6. I. Daubechies and J. C. Lagarias, Two-scale difference equations: II. Local regularity, infinite products of matrices and fractals, SIAM J. Math. Anal. 23 (1992), 1031-1079. MR 93g:39001

7. G. Donovan, J. S. Geronimo, D. P. Hardin, and P. R. Massopust, Construction of orthogonal wavelets using fractal interpolation functions, SIAM J. Math. Anal. 27(1996), 1158-1192. MR 97f: 42053

8. N. Dyn, J. A. Gregory, and D. Levin, Analysis of uniform binary subdivision schemes for curve design, Constr. Approx. 7 (1991), 127-147. MR 92d:65027

9. T. N. T. Goodman, R. Q. Jia, and C. A. Micchelli, On the spectral radius of a bi-infinite periodic and slanted matrix, Southeast Asian Bull. Math., to appear.

10. T. N. T. Goodman, C. A. Micchelli, and J. D. Ward, Spectral radius formulas for subdivision operators, in Recent Advances in Wavelet Analysis, L. L. Schumaker and G. Webb (eds.), Academic Press, 1994, pp. 335-360. MR 94m:47076

11. B. Han and R. Q. Jia, Multivariate refinement equations and subdivision schemes, SIAM J. Math. Anal., to appear.

12. C. Heil and D. Colella, Matrix refinement equations: existence and uniqueness, J. Fourier Anal. Appl. 2 (1996), 363-377. MR 97k:39021

13. C. Heil, G. Strang, and V. Strela, Approximation by translates of refinable functions, Numer. Math. 73 (1996), 75-94. MR 97c:65033

14. L. Hervé, Multi-resolution analysis of multiplicity d: Applications to dyadic interpolation, Applied and Computational Harmonic Analysis 1 (1994), 299-315. MR 97a:42026

15. T. A. Hogan, Stability and linear independence of the shifts of finitely many refinable functions, J. Fourier Anal. Appl. 3 (1997), 757-774. CMP 98:04

16. R. Q. Jia, Subdivision schemes in $L_{p}$ spaces, Advances in Comp. Math. 3 (1995), 309-341. MR 96d:65028

17. R. Q. Jia, Shift-invariant spaces on the real line, Proc. Amer. Math. Soc. 125 (1997), 785-793. MR 97e:41039

18. R. Q. Jia and C. A. Micchelli, On linear independence of integer translates of a finite number of functions, Proc. Edinburgh Math. Soc. 36 (1993), 69-85. MR 94e:41044

19. R. Q. Jia, S. Riemenschneider, and D. X. Zhou, Approximation by multiple refinable functions, Canadian J. Math. 49 (1997), 944-962.

20. R. Q. Jia and Z. W. Shen, Multiresolution and wavelets, Proc. Edinburgh Math. Soc. 37 (1994), 271-300. MR 95h:42035

21. R. Q. Jia and J. Z. Wang, Stability and linear independence associated with wavelet decompositions, Proc. Amer. Math. Soc. 117 (1993), 1115-1124. MR 93e:42046

22. W. Lawton, S. L. Lee, and Z. W. Shen, An algorithm for matrix extension and wavelet construction, Math. Comp. 214 (1996), 723-737. MR 96g:65018

23. W. Lawton, S. L. Lee, and Z. W. Shen, Stability and orthonormality of multivariate refinable functions, SIAM J. Math. Anal. 28 (1997), 999-1014. CMP 97:13

24. W. Lawton, S. L. Lee, and Z. W. Shen, Convergence of multidimensional cascade algorithm, Numer. Math. 78 (1998), 427-438.

25. R. L. Long, W. Chen, and S. L. Yuan, Wavelets generated by vector multiresolution analysis, Appl. Comput. Harmon. Anal. 4 (1997), no. 3, 293-316. CMP 97:14

26. R. L. Long and Q. Mo, $L^{2}$-convergence of vector cascade algorithm, manuscript.

27. C. A. Micchelli and H. Prautzsch, Uniform refinement of curves, Linear Algebra \& Appl. 114/115 (1989), 841-870. MR 90k:65088

28. G. Plonka, Approximation order provided by refinable function vectors, Constr. Approx. 13 (1997), 221-244. CMP 97:09

29. G.-C. Rota and G. Strang, A note on the joint spectral radius, Indag. Math. 22 (1960), 379-381. MR 26:5434

30. Z. W. Shen, Refinable function vectors, SIAM J. Math. Anal. 29 (1998), 235-250. 
31. L. F. Villemoes, Wavelet analysis of refinement equations, SIAM J. Math. Anal. 25 (1994), 1433-1460. MR 96f:39009

32. J. Z. Wang, Stability and linear independence associated with scaling vectors, SIAM J. Math. Anal., to appear

33. D. X. Zhou, Stability of refinable functions, multiresolution analysis and Haar bases, SIAM J. Math. Anal. 27 (1996), 891-904. MR 97h:42027

34. D. X. Zhou, Existence of multiple refinable distributions, Michigan Math. J. 44 (1997), 317329. CMP 97:15

(R.-Q. Jia and S. D. Riemenschneider) Department of Mathematical Sciences, University of Alberta, Edmonton, Canada T6G 2G1

E-mail address: jia@xihu.math.ualberta.ca

E-mail address: sherm@approx.math.ualberta.ca

(D.-X. Zhou) Department of Mathematics, City University of Hong Kong, Tat Chee Avenue, Kowloon, Hong Kong

E-mail address: mazhou@math.cityu.edu.hk 